\title{
GENERALIZED DESCENT ALGEBRA AND CONSTRUCTION OF IRREDUCIBLE CHARACTERS OF HYPEROCTAHEDRAL GROUPS
}

\author{
CÉDRIC BONNAFÉ AND CHRISTOPHE HOHLWEG
}

\section{INTRODUCTION}

Let $(W, S)$ be a finite Coxeter system and let $\ell: W \rightarrow \mathbb{N}$ denote the length function. If $I \subset S, W_{I}=<I>$ is the standard parabolic subgroup generated by $I$ and $X_{I}=\{w \in W \mid \forall s \in I, \ell(w s)>\ell(w)\}$ is a cross-section of $W / W_{I}$. Write $x_{I}=\sum_{w \in X_{I}} w \in \mathbb{Z} W$, then $\Sigma(W)=\oplus_{I \subset S} \mathbb{Z} x_{I}$ is a subalgebra of $\mathbb{Z} W$ and the $\mathbb{Z}$-linear map $\theta: \Sigma(W) \rightarrow \mathbb{Z} \operatorname{Irr} W, x_{I} \mapsto \operatorname{Ind}_{W_{I}}^{W} 1$ is a morphism of algebras: $\Sigma(W)$ is called the descent algebra or the Solomon algebra of $W$ [18. However, the morphism $\theta$ is surjective if and only if $W$ is a product of symmetric groups.

The aim of this paper is to construct, whenever $W$ is of type $C$, a subalgebra $\Sigma^{\prime}(W)$ of $\mathbb{Z} W$ containing $\Sigma(W)$ and a surjective morphism of algebras $\theta^{\prime}$ : $\Sigma^{\prime}(W) \rightarrow \mathbb{Z} \operatorname{Irr} W$ build similarly as $\Sigma(W)$ by starting with a bigger generating set. More precisely, let $\left(W_{n}, S_{n}\right)$ denote a Coxeter system of type $C_{n}$ and write $S_{n}=\left\{t, s_{1}, \ldots, s_{n-1}\right\}$ where the Dynkin diagram of $\left(W_{n}, S_{n}\right)$ is

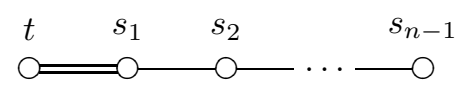

Let $t_{1}=t$ and $t_{i}=s_{i-1} t_{i-1} s_{i-1}(2 \leq i \leq n)$ and $S_{n}^{\prime}=S_{n} \cup\left\{t_{1}, \ldots, t_{n}\right\}$. Let $\mathcal{P}_{0}\left(S_{n}^{\prime}\right)$ denote the set of subsets $I$ of $S_{n}^{\prime}$ such that $I=<I>\cap S_{n}^{\prime}$. If $I \in \mathcal{P}_{0}\left(S_{n}^{\prime}\right)$, let $W_{I}, X_{I}$ and $x_{I}$ be defined as before. Then:

Theorem. $\Sigma^{\prime}\left(W_{n}\right)=\oplus_{I \in \mathcal{P}_{0}\left(S_{n}^{\prime}\right)} \mathbb{Z} x_{I}$ is a subalgebra of $\mathbb{Z} W_{n}$ and the $\mathbb{Z}$-linear map $\theta_{n}: \Sigma^{\prime}\left(W_{n}\right) \rightarrow \mathbb{Z} \operatorname{Irr} W_{n}, x_{I} \mapsto \operatorname{Ind}_{W_{I}}^{W} 1$ is a surjective morphism of algebras. Moreover, $\operatorname{Ker} \theta_{n}=\sum_{I \equiv I^{\prime}} \mathbb{Z}\left(x_{I}-x_{I^{\prime}}\right)$ and $\mathbb{Q} \otimes_{\mathbb{Z}} \operatorname{Ker} \theta_{n}$ is the radical of the $\mathbb{Q}$ algebra $\mathbb{Q} \otimes_{\mathbb{Z}} \Sigma^{\prime}\left(W_{n}\right)$.

In this theorem, the notation $I \equiv I^{\prime}$ means that there exists $w \in W_{n}$ such that $I^{\prime}={ }^{w} I$, that is, $W_{I}$ and $W_{I^{\prime}}$ are conjugated. This theorem is stated and proved in 3.3 Note that it is slightly differently formulated: in fact, it turns out that there is a natural bijection between signed compositions of $n$ and $\mathcal{P}_{0}\left(S_{n}^{\prime}\right)$ (see Lemma 2.5). So, everything in the text is indexed by signed compositions instead of $\mathcal{P}_{0}\left(S_{n}^{\prime}\right)$. It must also be noticed that, by opposition with the classical case, the multiplication $x_{I} x_{J}$ may involve negative coefficients. Using another basis, we show that $\Sigma^{\prime}\left(W_{n}\right)$ is precisely the generalized descent algebra discovered by Mantaci and Reutenauer [16.

Using this theorem and the Robinson-Schensted correspondence for type $C$ constructed by Stanley [20] and a Knuth version of it given in [5], we obtain an analog 
of Jöllenbeck's result (on the construction of characters of the symmetric group $\left[12\right.$ ) using an extension $\tilde{\theta}_{n}: \mathcal{Q}_{n} \rightarrow \mathbb{Z} \operatorname{Irr} W_{n}$ of $\theta_{n}$ to the coplactic space $\mathcal{Q}_{n}$ (see Theorem 4.14). The coplactic space refer to Jöllenbeck's construction revised in 3 .

Now, let $\mathcal{S P}=\oplus_{n \geq 0} \mathbb{Z} W_{n}, \Sigma^{\prime}=\oplus_{n \geq 0} \Sigma^{\prime}\left(W_{n}\right)$ and $\mathcal{Q}=\oplus_{n \geq 0} \mathcal{Q}_{n}$. Let $\theta=$ $\oplus_{n \geq 0} \theta_{n}$ and $\tilde{\theta}=\oplus_{n \geq 0} \tilde{\tilde{\theta}}_{n}$. Aguiar and Mahajan have proved that $\mathcal{S P}$ is naturally a Hopf algebra and that $\Sigma^{\prime}$ is a Hopf subalgebra [1]. We prove here that $\mathcal{Q}$ is also a Hopf subalgebra of $\mathcal{S P}$ (containing $\Sigma^{\prime}$ ) and that $\theta$ and $\tilde{\theta}$ are surjective morphisms of Hopf algebras (see Theorem [5.8). This generalizes similar results in symmetric groups (17] and [3]), which are parts of combinatorial tools used within the framework of the representation theory of type $A$ (see for instance [21]).

In the last section of this paper, we give some explicit computations in $\Sigma^{\prime}\left(W_{2}\right)$ (characters, complete set of orthogonal primitive idempotents, Cartan matrix of $\left.\Sigma^{\prime}\left(W_{2}\right) \ldots\right)$.

In the Appendix, P. Baumann and the second author link the above construction with the Specht construction and symmetric functions (see [14).

Remark. It seems interesting to try to construct a subalgebra $\Sigma^{\prime}(W)$ of $\mathbb{Z} W$ containing $\Sigma(W)$ and a morphism $\theta^{\prime}: \Sigma^{\prime}(W) \rightarrow \mathbb{Z} \operatorname{Irr} W$ for arbitrary Coxeter group $W$. But it is impossible to do so in a same way as we did for type $C$ (by extending the generating set). Computations using CHEVIE programs show us that it is impossible to do so in type $D_{4}$ and that the reasonable choices in $F_{4}$ fail (we do not obtain a subalgebra!). However, it is possible to do something similar for type $G_{2}$. More precisely, let $(W, S)$ be of type $G_{2}$. Write $S=\{s, t\}$ and let $S^{\prime}=\{s, t$, sts, tstst $\}$ and repeat the procedure described above to obtain a sub- $\mathbb{Z}$-module $\Sigma^{\prime}(W)$ of $\mathbb{Z} W$ and a morphism $\theta^{\prime}: \Sigma^{\prime}(W) \rightarrow \mathbb{Z} \operatorname{Irr} W$. Then the theorem stated in this introduction also holds in this case. We have $\operatorname{rank}_{\mathbb{Z}} \Sigma^{\prime}(W)=8$ and $\operatorname{rank}_{\mathbb{Z}} \operatorname{Ker} \theta^{\prime}=2$.

\section{Some REFLECTION SUbGROUPS OF HYPEROCTAHEDRAL GROUPS}

In this article, we denote $[m, n]=\{i \in \mathbb{Z} \mid m \leq i \leq n\}=\{m, m+1, \ldots, n-1, n\}$, for all $m \leq n \in \mathbb{Z}$, and $\operatorname{sign}(i) \in\{ \pm 1\}$ the $\operatorname{sign}$ of $i \in \mathbb{Z} \backslash\{0\}$. If $E$ is a set, we denote by $\mathfrak{S}(E)$ the group of permutations on the set $E$. If $m \in \mathbb{Z}$, we often denote by $\bar{m}$ the integer $-m$.

2.1. The hyperoctahedral group. We begin by making clear some notations and definitions concerning the hyperoctahedral group $W_{n}$. Denote $1_{n}$ the identity of $W_{n}$ (or 1 if no confusion is possible). We denote by $\ell_{t}(w)$ the number of occurrences of $t$ in a reduced decomposition of $w$ and we define $\ell_{s}(w)=\ell(w)-\ell_{t}(w)$.

It is well-known that $W_{n}$ acts on the set $I_{n}=[1, n] \cup[\bar{n}, \overline{1}]$ by permutations as follows: $t=(\overline{1}, 1)$ and $s_{i}=(\overline{i+1}, \bar{i})(i, i+1)$ for any $i \in[1, n-1]$. Through this action, we have

$$
W_{n}=\left\{w \in \mathfrak{S}\left(I_{n}\right) \mid \forall i \in I_{n}, w(\bar{i})=\overline{w(i)}\right\} .
$$

We often represent $w \in W_{n}$ as the word $w(1) w(2) \ldots w(n)$ in examples.

The subgroup $W_{\bar{n}}=\left\{w \in W_{n} \mid w([1, n])=[1, n]\right\}$ of $W_{n}$ is naturally identified with $\mathfrak{S}_{n}$, the symmetric group of degree $n$, by restriction of its elements to $[1, n]$. Note that $W_{\bar{n}}$ is generated, as a reflection subgroup of $W_{n}$, by $S_{\bar{n}}=\left\{s_{1}, \ldots, s_{n-1}\right\}$. 
A standard parabolic subgroup of $W_{n}$ is a subgroup generated by a subset of $S_{n}$ (a parabolic subgroup of $W_{n}$ is a subgroup conjugate to some standard parabolic subgroup). Note that $\left(W_{\bar{n}}, S_{\bar{n}}\right)$ is a Coxeter group, which is a standard parabolic subgroup of $W_{n}$. If $m \leq n$, then $S_{m}$ is naturally identified with a subset of $S_{n}$ and $W_{m}$ will be identified with the standard parabolic subgroup of $W_{n}$ generated by $S_{m}$.

Now, we set $T_{n}=\left\{t_{1}, \ldots, t_{n}\right\}$, with $t_{i}$ as in Introduction. As a permutation of $I_{n}$, note that $t_{i}=(i, \bar{i})$, then the reflection subgroup $\mathfrak{T}_{n}$ generated by $T_{n}$ is naturally identified with $(\mathbb{Z} / 2 \mathbb{Z})^{n}$. Therefore $W_{n}=W_{\bar{n}} \ltimes \mathfrak{T}_{n}$ is just the wreath product of $\mathfrak{S}_{n}$ by $\mathbb{Z} / 2 \mathbb{Z}$. If $w \in W_{n}$, we denote by $\left(w_{S}, w_{T}\right)$ the unique pair in $\mathfrak{S}_{n} \times \mathfrak{T}_{n}$ such that $w=w_{S} w_{T}$. Note that $\ell_{t}(w)=\ell_{t}\left(w_{T}\right)$. In this article, we will consider reflection subgroups generated by subsets of $S_{n}^{\prime}=S_{n} \cup T_{n}$.

2.2. Root system. Before studying the reflection subgroups generated by subsets of $S_{n}^{\prime}$, let us recall some basic facts about Weyl groups of type $C$ (see [6]). Let us endow $\mathbb{R}^{n}$ with its canonical euclidean structure. Let $\left(e_{1}, \ldots, e_{n}\right)$ denote the canonical basis of $\mathbb{R}^{n}$ : this is an orthonormal basis. If $\alpha \in \mathbb{R}^{n} \backslash\{0\}$, we denote by $s_{\alpha}$ the orthogonal reflection such that $s_{\alpha}(\alpha)=-\alpha$. Let

$$
\Phi_{n}^{+}=\left\{2 e_{i} \mid 1 \leq i \leq n\right\} \cup\left\{e_{j}+\nu e_{i} \mid \nu \in\{1,-1\} \text { and } 1 \leq i<j \leq n\right\},
$$

$\Phi_{n}^{-}=-\Phi_{n}^{+}$and $\Phi_{n}=\Phi_{n}^{+} \cup \Phi_{n}^{-}$. Then $\Phi_{n}$ is a root system of type $C_{n}$ and $\Phi_{n}^{+}$is a positive root system of $\Phi_{n}$. By sending $t$ to $s_{2 e_{1}}$ and $s_{i}$ to $s_{e_{i+1}-e_{i}}$ (for $1 \leq i \leq n-1$ ), we will identify $W_{n}$ with the Coxeter group of $\Phi_{n}$. Then

$$
\Delta_{n}=\left\{2 e_{1}, e_{2}-e_{1}, e_{3}-e_{2}, \ldots, e_{n}-e_{n-1}\right\}
$$

is the basis of $\Phi_{n}$ contained in $\Phi_{n}^{+}$and the subset $S_{n}$ of $W_{n}$ is naturally identified with the set of simple reflections $\left\{s_{\alpha} \mid \alpha \in \Delta_{n}\right\}$. Therefore, for any $w \in W_{n}$ we have

$$
\ell(w)=\left|\Phi_{n}^{+} \cap w^{-1}\left(\Phi_{n}^{-}\right)\right| ;
$$

and $\ell\left(w s_{\alpha}\right)<\ell(w)$ if and only if $w(\alpha) \in \Phi^{-}$, for all $\alpha \in \Phi^{+}$.

Remark 2.1 - Let $w \in W_{n}$ and let $\alpha \in \Phi_{n}^{+}$. Then $\ell\left(w s_{\alpha}\right)<\ell(w)$ if and only if $w(\alpha) \in \Phi_{n}^{-}$. Therefore, if $i \in[1, n-1]$, then

$$
\ell\left(w s_{i}\right)<\ell(w) \Leftrightarrow w(i)>w(i+1),
$$

and, if $j \in[1, n]$, then

$$
\ell\left(w t_{j}\right)<\ell(w) \Leftrightarrow w(j)<0 .
$$

Therefore, we deduce from the strong exchange condition (see [11, §5.8])

$$
\ell_{t}(w)=|\{i \in[1, n] \mid w(i)<0\}| .
$$

2.3. Some closed subsystems of $\Phi_{n}$. Consider the subsets $\left\{s_{1}, t_{1}\right\}$ and $\left\{s_{1}, t_{2}\right\}$ of $S_{n}^{\prime}(n \geq 2)$. It is readily seen that these two sets of reflections generate the same reflection subgroup of $W_{n}$. This lead us to find a parametrization of subgroups generated by a subset of $S_{n}^{\prime}$.

A signed composition is a sequence $C=\left(c_{1}, \ldots, c_{r}\right)$ of non-zero elements of $\mathbb{Z}$. The number $r$ is called the length of $C$. We set $|C|=\sum_{i=1}^{r}\left|c_{i}\right|$. If $|C|=n$, we say that $C$ is a signed composition of $n$ and we write $C \|=n$. We also define $C^{+}=\left(\left|c_{1}\right|, \ldots,\left|c_{r}\right|\right) \| n, C^{-}=-C^{+}$and $\bar{C}=-C$. We denote by $\operatorname{Comp}(n)$ the set 
of signed compositions of $n$. In particular, any composition is a signed composition (any part is positive). Note that

$$
|\operatorname{Comp}(n)|=2.3^{n-1} \text {. }
$$

Now, to each $C=\left(c_{1}, \ldots, c_{r}\right) \| n$, we associate a reflection subgroup of $W_{n}$ as follows: for $1 \leq i \leq r$, set

$$
I_{C}^{(i)}= \begin{cases}I_{C,+}^{(i)} & \text { if } c_{i}<0, \\ I_{C,+}^{(i)} \cup-I_{C,+}^{(i)} & \text { if } c_{i}>0,\end{cases}
$$

where $I_{C,+}^{(i)}=\left[\left|c_{1}\right|+\cdots+\left|c_{i-1}\right|+1,\left|c_{1}\right|+\cdots+\left|c_{i}\right|\right]$. Then

$$
W_{C}=\left\{w \in W_{n} \mid \forall 1 \leq i \leq r, w\left(I_{C}^{(i)}\right)=I_{C}^{(i)}\right\}
$$

is a reflection subgroup generated by

$$
\begin{aligned}
S_{C}= & \left\{s_{p} \in S_{\bar{n}}|| c_{1}|+\cdots+| c_{i-1}|+1 \leq p \leq| c_{1}|+\cdots+| c_{i} \mid-1\right\} \\
& \cup\left\{t_{\left|c_{1}\right|+\cdots+\left|c_{j-1}\right|+1} \in T_{n} \mid c_{j}>0\right\} \subset S_{n}^{\prime}
\end{aligned}
$$

Therefore, $W_{C} \simeq W_{c_{1}} \times \cdots \times W_{c_{r}}$ : we denote by $\left(w_{1}, \ldots, w_{r}\right) \mapsto w_{1} \times \cdots \times w_{r}$ the natural isomorphism $W_{c_{1}} \times \cdots \times W_{c_{r}} \stackrel{\sim}{\longrightarrow} W_{C}$.

Example. The group $W_{(\overline{2}, 3, \overline{1}, \overline{3}, 1)} \simeq \mathfrak{S}_{2} \times W_{3} \times \mathfrak{S}_{1} \times \mathfrak{S}_{3} \times W_{1}$ is generated, as a reflection subgroup of $W_{10}$, by $S_{(\overline{2}, 3, \overline{1}, \overline{3}, 1)}=\left\{s_{1}\right\} \cup\left\{t_{3}, s_{3}, s_{4}\right\} \cup\left\{s_{7}, s_{8}\right\} \cup\left\{t_{10}\right\} \subset S_{10}^{\prime}$.

The signed composition $C$ is said semi-positive if $c_{i} \geq-1$ for every $i \in[1, r]$. Note that a composition is a semi-positive composition. We say that $C$ is negative if $c_{i}<0$ for every $i \in[1, r]$. We say that $C$ is parabolic if $c_{i}<0$ for $i \in[2, r]$. Note that $C$ is parabolic if and only if $W_{C}$ is a standard parabolic subgroup.

Now, let $S_{C}^{\prime}=S_{n}^{\prime} \cap W_{C}, \Phi_{C}=\left\{\alpha \in \Phi_{n} \mid s_{\alpha} \in W_{C}\right\}$ and $\Phi_{C}^{+}=\Phi_{C} \cap \Phi_{n}^{+}$. Then $W_{C}$ is the Weyl group of the closed subsystem $\Phi_{C}$ of $\Phi_{n}$. Moreover, $\Phi_{C}^{+}$is a positive root system of $\Phi_{C}$ and we denote by $\Delta_{C}$ the basis of $\Phi_{C}$ contained in $\Phi_{C}^{+}$. Note that $S_{C}=\left\{s_{\alpha} \mid \alpha \in \Delta_{C}\right\}$, so $\left(W_{C}, S_{C}\right)$ is a Coxeter group.

Let $\ell_{C}: W_{C} \rightarrow \mathbb{N}$ denote the length function on $W_{C}$ with respect to $S_{C}$. Let $w_{C}$ denote the longest element of $W_{C}$ with respect to $\ell_{C}$. If $C$ is a composition, we denote by $\sigma_{C}$ the longest element of $\mathfrak{S}_{C}=W_{\bar{C}}$ with respect to $\ell_{\bar{C}}$ (which is the restriction of $\ell$ to $\mathfrak{S}_{C}$ ). In other words, $\sigma_{C}=w_{\bar{C}}$. In particular, $w_{n}\left(\right.$ resp. $\left.\sigma_{n}\right)$ denotes the longest element of $W_{n}$ (resp. $\left.\mathfrak{S}_{n}\right)$.

Write $T_{C}=T_{n} \cap W_{C}$ and $\mathfrak{T}_{C}=\mathfrak{T}_{n} \cap W_{C}$, then observe that

$$
W_{C}=W_{C^{-}} \ltimes \mathfrak{T}_{C}=\mathfrak{S}_{C^{+}} \ltimes \mathfrak{T}_{C} .
$$

Remarks. (1) This class of reflection subgroups contains the standard parabolic subgroups, since $S_{n} \subset S_{n}^{\prime}$. But it contains also some other subgroups which are not parabolic (consider the subgroup generated by $\left\{t_{1}, t_{2}\right\}$ as example). In other words, it may happen that $\Delta_{C} \not \subset \Delta_{n}$. In fact, $\Delta_{C} \subset \Delta_{n}$ if and only if $W_{C}$ is a standard parabolic subgroup of $W_{n}$.

(2) If $W_{C}$ is not a standard parabolic subgroup of $W_{n}$, then $\ell_{C}$ is not the restriction of $\ell$ to $W_{C}$.

We close this subsection by an easy characterization of the subsets $S_{C}^{\prime}$ :

Lemma 2.5. Let $X$ be a subset of $S_{n}^{\prime}$. Then the following are equivalent: 
(1) $<X>\cap S_{n}^{\prime}=X$.

(2) $X \cap T_{n}$ is stable under conjugation by $\langle X>$.

(3) $X \cap T_{n}$ is stable under conjugation by $<X \cap S_{\bar{n}}>$.

(4) There exists a signed composition $C$ of $n$ such that $X=S_{C}^{\prime}$.

Corollary 2.6. Let $w \in W_{n}$ and let $C \| n$. If ${ }^{w} S_{C}^{\prime} \subset S_{n}^{\prime}$, then there exists a (unique) signed composition $D$ such that ${ }^{w} S_{C}^{\prime}=S_{D}^{\prime}$.

Proof. Indeed, ${ }^{w} S_{C}^{\prime} \cap T_{n}={ }^{w}\left(S_{C}^{\prime} \cap T_{n}\right)$ and ${ }^{w} S_{C}^{\prime} \cap S_{\bar{n}}={ }^{w}\left(S_{C}^{\prime} \cap S_{\bar{n}}\right)$.

2.4. Orbits of closed subsystems of $\Phi_{n}$. In this subsection, we determine when two subgroups $W_{C}$ and $W_{D}$ of $W_{n}$ are conjugated. A bipartition of $n$ is a pair $\lambda=\left(\lambda^{+}, \lambda^{-}\right)$of partitions such that $|\lambda|:=\left|\lambda^{+}\right|+\left|\lambda^{-}\right|=n$. We write $\lambda \Vdash n$ to say that $\lambda$ is a bipartition of $n$, and the set of bipartitions of $n$ is denoted by $\operatorname{Bip}(n)$. It is well-known that the conjugacy classes of $W_{n}$ are in bijection with $\operatorname{Bip}(n)$ (see 9] [14]). We define $\hat{\lambda}$ as the signed composition of $n$ obtained by concatenation of $\lambda^{+}$and $-\lambda^{-}$. The $\operatorname{map} \operatorname{Bip}(n) \rightarrow \operatorname{Comp}(n), \lambda \mapsto \hat{\lambda}$ is injective.

Now, let $C$ be a signed composition of $n$. We define $\boldsymbol{\lambda}(C)=\left(\lambda^{+}, \lambda^{-}\right)$as the bipartition of $n$ such that $\lambda^{+}$(resp. $\lambda^{-}$) is obtained from $C$ by reordering in decreasing order the positive parts of $C$ (resp. the absolute value of the negative parts of $C$ ). One can easily check that the map

$$
\boldsymbol{\lambda}: \operatorname{Comp}(n) \longrightarrow \operatorname{Bip}(n)
$$

is surjective (indeed, if $\lambda \in \operatorname{Bip}(n)$, then $\boldsymbol{\lambda}(\hat{\lambda})=\lambda)$ and that the following proposition holds:

Proposition 2.7. Let $C, D \| n$, then $W_{C}$ and $W_{D}$ are conjugate in $W_{n}$ if and only if $\boldsymbol{\lambda}(C)=\boldsymbol{\lambda}(D)$. If $\Psi$ is a closed subsystem of $\Phi_{n}$, then there exists a unique bipartition $\lambda$ of $n$ and some $w \in W_{n}$ such that $\Psi=w\left(\Phi_{\hat{\lambda}}\right)$.

Let $C, D \| n$, then we write $C \subset D$ if $W_{C} \subset W_{D}$. Moreover, $C, C^{\prime} \subset D$ and if $W_{C}$ and $W_{C^{\prime}}$ are conjugate under $W_{D}$, then we write $C \equiv_{D} C^{\prime}$.

2.5. Distinguished coset representatives. Let $C \| n$, then

$$
X_{C}=\left\{x \in W_{n} \mid \forall w \in W_{C}, \ell(x w) \geq \ell(x)\right\}
$$

is a distinguished set of minimal coset representatives for $W_{n} / W_{C}$ (see proposition below). It is readily seen that

$$
\begin{aligned}
X_{C} & =\left\{w \in W_{n} \mid w\left(\Phi_{C}^{+}\right) \subset \Phi_{n}^{+}\right\} \\
& =\left\{w \in W_{n} \mid \forall \alpha \in \Delta_{C}, w(\alpha) \in \Phi_{n}^{+}\right\} .
\end{aligned}
$$

Finally

$$
X_{C}=\left\{w \in W_{n} \mid \forall r \in S_{C}, \ell(w r)>\ell(w)\right\} .
$$

We need a relative notion: if $D \| n$ such that $C \subset D$, the set $X_{C}^{D}=X_{C} \cap W_{D}$ is a distinguished set of minimal coset representatives for $W_{D} / W_{C}$. If $D=(n)$ we write $X_{C}^{n}$ instead of $X_{C}^{(n)}$.

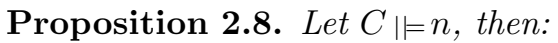

(a) The map $X_{C} \times W_{C} \rightarrow W_{n},(x, w) \mapsto x w$ is bijective.

(b) If $C \subset D$, then the map $X_{D} \times X_{C}^{D} \rightarrow X_{C},(x, y) \mapsto x y$ is bijective.

(c) If $x \in X_{C}$ and $w \in W_{C}$, then $\ell_{t}\left(x w x^{-1}\right) \geq \ell_{t}(w)$. Consequently, $\mathfrak{S}_{n} \cap$ ${ }^{x} W_{C}=\mathfrak{S}_{n} \cap{ }^{x} \mathfrak{S}_{C^{+}}$. 
Proof. (a) is stated, in a general case, in [7 Proposition 3.1]. (b) follows easily from (a). Let us now prove (c). Let $x \in X_{C}$ and $w \in W_{C}$. Let $I=\left\{i \in I_{n} \mid w(i)<0\right\}$ and $J=\left\{i \in I_{n} \mid x w x^{-1}(i)<0\right\}$, then $\ell_{t}(w)=|I|$ and $\ell_{t}\left(x w x^{-1}\right)=|J|$, by 2.2 , Now let $i \in I$, then $t_{i} \in W_{C}$, so $\ell_{t}\left(x t_{i}\right)>\ell_{t}(x)$. In other words, $x(i)>0$. Now, we have $x w x^{-1}(x(i))=x w(i)$. But, $w(i)<0$ and $t_{-w(i)}=w t_{i} w^{-1} \in W_{C}$. Therefore, $x(-w(i))=-x w(i)>0$. This shows that $x(i) \in J$. So, the map $I \rightarrow J, i \mapsto x(i)$ is well-defined and clearly injective, implying $|I| \leq|J|$ as desired.

The last assertion of this proposition follows easily from this inequality and from the fact that $\mathfrak{S}_{C^{+}}=\left\{w \in W_{C} \mid \ell_{t}(w)=0\right\}$.

Proposition 2.9. Let $C \| n$ and $x \in X_{C}$ be such that ${ }^{x} S_{C}^{\prime} \subset S_{n}^{\prime}$. Let $D$ be the unique signed composition of $n$ such that ${ }^{x} S_{C}^{\prime}=S_{D}^{\prime}$ (see Corollary 2.6). Then $X_{C}=X_{D} x$.

Proof. By symmetry, it is sufficient to prove that, if $w \in X_{D}$, then $w x \in X_{C}$. Let $\alpha \in \Phi_{C}^{+}$. Then, since $x \in X_{C}$, we have $x(\alpha) \in \Phi_{n}^{+} \cap{ }^{x} \Phi_{C}=\Phi_{D}^{+}$. So $w(x(\alpha)) \in \Phi_{n}^{+}$ since $w \in X_{D}$. So $w x \in X_{C}$.

2.6. Maximal element in $X_{C}$. It turns out that, for every signed composition $C$ of $n, X_{C}$ contains a unique element of maximal length (see Proposition 2.12). First, note the following two examples:

(1) if $C$ is parabolic, it is well-known that $\ell_{C}$ is the restriction of $\ell$ and that, for all $(x, w) \in X_{C} \times W_{C}$, we have

$$
\ell(x w)=\ell(x)+\ell(w)
$$

In particular, $w_{n} w_{C}$ is the longest element of $X_{C}$ (see [9]);

(2) let $C$ be a composition of $n$, then $W_{C}$ is not in general a standard parabolic subgroup of $W_{n}$. However, since $W_{C}$ contains $\mathfrak{T}_{n}, X_{C}$ is contained in $\mathfrak{S}_{n}$. This shows that

$$
X_{C}=X_{\bar{C}}^{\bar{n}}=X_{\bar{C}} \cap \mathfrak{S}_{n} .
$$

In particular, $X_{C}$ contains a unique element of maximal length: this is $\sigma_{n} \sigma_{C}$;

Now, let $k$ and $l$ be two non-zero natural numbers such that $k+l=n$. Then $W_{k, l}$ is not a parabolic subgroup of $W_{n}$. However, $W_{k, \bar{l}}$ is a standard parabolic subgroup of $W_{n}$ and $W_{k, \bar{l}} \subset W_{k, l}$. So $X_{k, l} \subset X_{k, \bar{l}}$. So, if $x \in X_{k, l}$ and $w \in W_{k, \bar{l}}$, then

$$
\ell(x w)=\ell(x)+\ell(w) .
$$

This applies for instance if $w \in W_{k} \subset W_{k, \bar{l}}$.

Then, we need to introduce a decomposition of $X_{C}$ using Proposition 2.8 (b). Write $C=\left(c_{1}, \ldots, c_{r}\right) \| n$. We set

$$
X_{C, i}=X_{\left(\left|c_{1}\right|+\cdots+\left|c_{i-1}\right|, c_{i}, \ldots, c_{r}\right)}^{\left(\left|c_{1}\right|+\cdots+\left|c_{i}\right|, c_{i+1}, \ldots, c_{r}\right)}
$$

Then the map

$$
\begin{array}{ccc}
X_{C, r} \times \cdots \times X_{C, 2} \times X_{C, 1} & \longrightarrow & X_{C} \\
\left(x_{r}, \ldots, x_{2}, x_{1}\right) & \longmapsto & x_{r} \ldots x_{2} x_{1}
\end{array}
$$

is bijective by Proposition 2.8 (b). Moreover, by 2.10 we have

$$
\ell\left(x_{r} \ldots x_{2} x_{1}\right)=\ell\left(x_{r}\right)+\cdots+\ell\left(x_{2}\right)+\ell\left(x_{1}\right)
$$


for every $\left(x_{r}, \ldots, x_{2}, x_{1}\right) \in X_{C, r} \times \cdots \times X_{C, 2} \times X_{C, 1}$. For every $i \in[1, r], X_{C, i}$ contains a unique element of maximal length (see (1)-(2) above). Let us denote it by $\eta_{C, i}$. We set:

$$
\eta_{C}=\eta_{C, r} \ldots \eta_{C, 2} \eta_{C, 1}
$$

Then, by 2.11] we have

Proposition 2.12. Let $C \| n$, then $\eta_{C}$ is the unique element of $X_{C}$ of maximal length.

2.7. Double cosets representatives. If $C$ and $D$ are two signed compositions of $n$, we set

$$
X_{C D}=X_{C}^{-1} \cap X_{D}
$$

Proposition 2.13. Let $C$ and $D$ be two signed composition of $n$ and let $d \in X_{C D}$. Then:

(a) There exists a unique signed composition $E$ of $n$ such that $S_{E}^{\prime}=S_{C}^{\prime} \cap{ }^{d} S_{D}^{\prime}$. It will be denoted by $C \cap{ }^{d} D$ or ${ }^{d} D \cap C$. We have $\left(C \cap{ }^{d} D\right)^{-}=C^{-} \cap{ }^{d} D^{-}$.

(b) $W_{C} \cap{ }^{d} W_{D}=W_{C \cap{ }^{d} D}$ and $W_{C} \cap{ }^{d} S_{D}^{\prime}=S_{C}^{\prime} \cap{ }^{d} W_{D}=S_{C \cap^{d} D}^{\prime}$.

(c) If $w \in W_{C \cap^{d} D}$, then $\ell_{t}(w)=\ell_{t}\left(d^{-1} w d\right)$.

(d) If $w \in W_{C} d W_{D}$, then there exists a unique pair $(x, y) \in X_{C \cap^{d} D}^{C} \times W_{D}$ such that $w=x d y$.

(e) Let $(x, y) \in X_{C \cap^{d} D}^{C} \times W_{D}$, then $\ell(x d y) \geq \ell\left(x_{S}\right)+\ell_{t}(x)+\ell(d)+\ell\left(y_{S}\right)+\ell_{t}(y)$.

(f) $d$ is the unique element of $W_{C} d W_{D}$ of minimal length.

Proof. (a) follows immediately from Lemma 2.5 (equivalence between (3) and (4)).

(b) It is clear that $W_{E} \subset W_{C} \cap{ }^{d} W_{D}$. Let us show the reverse inclusion. Let $w \in W_{C} \cap{ }^{d} W_{D}$. We will show by induction on $\ell_{t}(w)$ that $w \in W_{E}$. If $\ell_{t}(w)=0$, then we see from Proposition 2.8 (d) that $w \in \mathfrak{S}_{C^{+}} \cap{ }^{d} \mathfrak{S}_{D^{+}}=\mathfrak{S}_{E^{+}}$by definition of $E^{+}$.

Assume now that $\ell_{t}(w)>0$ and that, if $w^{\prime} \in W_{C} \cap{ }^{d} W_{D}$ is such that $\ell_{t}\left(w^{\prime}\right)<$ $\ell_{t}(w)$, then $w^{\prime} \in W_{E}$. Since $\ell_{t}(w)>0$, there exists $i \in[1, n]$ such that $w(i)<0$. In particular, $t_{i} \in \mathfrak{T}_{C}$. By the same argument as in the proof of Proposition 2.8 (d), we have that $t_{i} \in{ }^{d} W_{D}$. So, $t_{i} \in T_{C} \cap{ }^{d} T_{D}=T_{E}$. Now, let $w^{\prime}=w t_{i}$. Then $t_{i} \in W_{E}, w^{\prime} \in W_{C} \cap{ }^{d} W_{D}$ and $\ell_{t}\left(w^{\prime}\right)=\ell_{t}(w)-1$. So, by the induction hypothesis, $w^{\prime} \in W_{E}$, so $w \in W_{E}$.

The other assertions of (b) follow easily.

(c) Let $w=\sigma_{1} \ldots \sigma_{l}$ be a reduced decomposition of $w$ with respect to $S_{C}$. Then $d^{-1} w d=\left(d^{-1} \sigma_{1} d\right) \ldots\left(d^{-1} \sigma_{l} d\right)$. But $d^{-1} \sigma_{i} d \in d^{-1}\left(S_{C}^{\prime} \cap{ }^{d} S_{D}^{\prime}\right)=S_{d^{-1} C \cap D}^{\prime}$, so $\ell_{t}\left(d^{-1} \sigma_{i} d\right)=\ell_{t}\left(\sigma_{i}\right)$. Since $\ell_{t}(w)=\ell_{t}\left(\sigma_{1}\right)+\cdots+\ell_{t}\left(\sigma_{l}\right)$, we see that $\ell_{t}(w) \geq$ $\ell_{t}\left(d^{-1} w d\right)$. By symmetry, we obtain the reverse inequality.

(d) Let $w \in W_{C} d W_{D}$. Let us write $w=a d b$, with $a \in W_{C}$ and $b \in W_{D}$. We then write $a=x a^{\prime}$ with $x \in X_{C \cap{ }^{d} D}^{C}$ and $a^{\prime} \in \mathfrak{S}_{C \cap^{d} D}$. Then $d^{-1} a^{\prime} d \in W_{d^{-1} C \cap D} \subset W_{D}$. Write $y=\left(d^{-1} a^{\prime} d\right) b$. Then $(x, y) \in X_{C \cap^{d} D}^{C} \times W_{D}$ and $w=x d y$.

Now let $\left(x^{\prime}, y^{\prime}\right) \in X_{C \cap^{d} D}^{C} \times W_{D}$ such that $w=x^{\prime} d y^{\prime}$. Then $x^{\prime-1} x=d\left(y y^{\prime-1}\right) d^{-1}$. So $x^{-1} x \in W_{C \cap^{d} D}$, that is $x W_{C}=x^{\prime} W_{C}$. So $x=x^{\prime}$ and $y=y^{\prime}$.

(e) Let $(x, y) \in X_{C \cap^{d} D}^{C} \times W_{D}$. We will show by induction on $\ell_{t}(x)+\ell_{t}(y)$ that

$$
\ell(x d y) \geq \ell\left(x_{S}\right)+\ell_{t}(x)+\ell(d)+\ell\left(y_{S}\right)+\ell_{t}(y) .
$$


If $\ell_{t}(x)=\ell_{t}(y)=0$, then $x \in X_{C^{-} \cap^{-}\left(D^{-}\right)}^{C^{-}}, y \in \mathfrak{S}_{D^{+}}$and $d \in X_{C^{-}, D^{-}}$. So, by [2, Lemma 2], we have $\ell(x d y)=\ell\left(x_{S}\right)+\ell(d)+\ell\left(y_{S}\right)$, as desired.

Now, let us assume that $\ell_{t}(x)+\ell_{t}(y)>0$ and that the result holds for every pair $\left(x^{\prime}, y^{\prime}\right) \in X_{C \cap^{d} D}^{C} \times W_{D}$ such that $\ell_{t}\left(x^{\prime}\right)+\ell_{t}\left(y^{\prime}\right)<\ell_{t}(x)+\ell_{t}(y)$. By symmetry, and using (c), we can assume that $\ell_{t}(y)>0$. So there exists $i \in I_{n}$ such that $y(i)<0$. Let $y^{\prime}=y t_{i}$. Then $t_{i} \in T_{D}, \ell\left(y_{S}\right)=\ell\left(y_{S}^{\prime}\right), \ell_{t}\left(y^{\prime}\right)=\ell_{t}(y)-1$. Therefore, by induction hypothesis, we have

$$
\ell\left(x d y^{\prime}\right) \geq \ell\left(x_{S}\right)+\ell_{t}(x)+\ell(d)+\ell\left(y_{S}\right)+\ell_{t}(y)-1 .
$$

It is now enough to show that $\ell\left(x d y^{\prime} t_{i}\right)>\ell\left(x d y^{\prime}\right)$, that is $x d y^{\prime}(i)>0$. Note that $y^{\prime}(i)>0$ and that $t_{y^{\prime}(i)}=y^{\prime} t_{i} y^{\prime-1} \in W_{D}$. So the result follows from the following lemma:

Lemma 2.14. If $d \in X_{C D}$, if $x \in X_{C \cap{ }^{d} D}^{D}$ and if $j \in[1, n]$ is such that $t_{j} \in T_{D}$, then $x d(j)>0$.

Proof. Since $t_{j} \in W_{D}$ and $d \in X_{D}$, we have $d(j)>0$. Two cases may occur. If $t_{d(j)} \in T_{C}$, then $t_{d(j)}=d t_{j} d^{-1} \in T_{C \cap^{d} D}$. Therefore, $x(d(j))>0$ since $x \in X_{C \cap^{d} D}^{C}$. If $t_{d(j)} \notin T_{C}$, then $x(d(j))>0$ since $x \in W_{C}=\mathfrak{S}_{C^{+}} \ltimes \mathfrak{T}_{C}$.

(f) follows immediately from (e).

Remark 2.15 - Let $C$ and $D$ be two signed compositions of $n$ and let $d \in X_{C D}$. Then $d^{-1} \in X_{D C}$ and, by Proposition 2.9 we have that

$$
X_{C \cap{ }^{d} D} d=X_{d^{-1} C \cap D}
$$

Corollary 2.16. The map $X_{C D} \rightarrow W_{C} \backslash W_{n} / W_{D}$ is bijective.

Proof. The proposition 2.13 (f) shows that the map is injective. The surjectivity follows from the fact that, if $w \in W_{n}$ is an element of minimal length in $W_{C} w W_{D}$, then $w \in X_{C D}$.

Corollary 2.17. If $C$ is parabolic or if $D$ is semi-positive, then

$$
X_{D}=\coprod_{d \in X_{C D}} X_{C \cap^{d} D}^{C} d .
$$

Proof. It follows from Corollary 2.16 that

$$
\left|X_{D}\right|=\left|W_{n} / W_{D}\right|=\sum_{d \in X_{C D}}\left|W_{C} d W_{D} / W_{D}\right|=\sum_{d \in X_{C D}}\left|X_{C \cap^{d} D}\right|,
$$

the last equality following from Proposition 2.13 (d). So, it remains to show that, if $d \in X_{C D}$ and if $x \in X_{C \cap^{d} D}^{C}$, then $x d \in X_{D}$.

Assume that we have found $s \in S_{D}$ such that $\ell(x d s)<\ell(x d)$. If $s \in T_{D}$, then $s=$ $t_{i}$ for som $i \in I_{n}$. But, by Lemma 2.14 $x d(i)>0$, so $\ell\left(x d t_{i}\right)>\ell(x d)$, contradicting our hypothesis. Therefore, $s \in S_{D^{-}}$, that is $s=s_{i}$ for some $i \in[1, n-1]$. If $C$ is parabolic, $C \cap{ }^{d} D$ is also parabolic. Therefore, $\ell(x d s)>\ell(x d)$ which is a contradiction, so $D$ is semi-positive. Therefore, we have that $t_{i}$ and $t_{i+1}$ belong to 
$T_{D}$. Thus, by Lemma 2.14 we have $x d(i)>0$ and $x d(i+1)>0$. Moreover, since $\ell\left(x d s_{i}\right)<\ell(x d)$, we have

$$
0<x d(i+1)<x d(i)
$$

But, since $d \in X_{D}$, we have $d(i+1)>d(i)$. So, by Proposition 2.13 (b), we have that $d s_{i} d^{-1} \in S_{C \cap^{d} D}$. Thus $\ell\left(x\left(d s_{i} d^{-1}\right)\right)>\ell(x)$ because $x \in X_{C \cap^{d} D}^{C}$. In other words, $x d(i+1)>x d(i)$. This contradicts $(*)$.

If $E$ is a signed composition of $n$ such that $C \subset E$ and $D \subset E$, we set $X_{C D}^{E}=$ $X_{C D} \cap W_{E}$.

Example. It is not true in general that $X_{D}=\coprod_{d \in X_{C D}} X_{C \cap^{d} D}^{C} d$. This is false, if $n=k+l$ with $k, l \geq 1, C=(\bar{k}, \bar{l})$ and $D=(n)$. See Example 2.25 for precisions.

In [2], the authors has given a proof of the Solomon theorem using tools which sound like the above results. Here, we cannot translate their proof because of the complexity of the decomposition of $X_{D}$ (which involve negative coefficients).

2.8. A partition of $\boldsymbol{W}_{\boldsymbol{n}}$. If $C=\left(c_{1}, \ldots, c_{r}\right)$ is a signed composition of $n$, we set

$$
A_{C}=\left\{s_{\left|c_{1}\right|+\cdots+\left|c_{i}\right|} \mid i \in[1, r] \text { and } c_{i}<0 \text { and } c_{i+1}>0\right\}
$$

and

$$
\mathcal{A}_{C}=S_{C}^{\prime} \coprod A_{C} .
$$

As example, $A_{(1, \overline{3}, \overline{1}, 2, \overline{1}, 1)}=\left\{s_{5}, s_{8}\right\}$. Note that $\mathcal{A}_{C}=\mathcal{A}_{D}$ if and only if $C=D$. If $w \in W_{n}$, then we define the ascent set of $w$ :

$$
\mathcal{U}_{n}^{\prime}(w)=\left\{s \in S_{n}^{\prime} \mid \ell(w s)>\ell(w)\right\} .
$$

Finally, following Mantaci-Reutenauer, we associate to each element $w \in W_{n}$ a signed composition $\boldsymbol{C}(w)$ as follows. First, let $\boldsymbol{C}^{+}(w)$ denote the biggest composition (for the order $\subset$ ) of $n$ such that, for every $1 \leq i \leq r$, the map $w: I_{C^{+}(w)}^{(i)} \rightarrow I_{n}$ is increasing and has constant sign. Now, we define $\nu_{i}=\operatorname{sign}(w(j))$ for $j \in I_{\boldsymbol{C}^{+}(w)}^{(i)}$. The descent composition of $w$ is $\boldsymbol{C}(w)=\left(\nu_{1} c_{1}^{+}, \ldots, \nu_{r} c_{r}^{+}\right)$.

Example. $\boldsymbol{C}(\underbrace{9 . \overline{3} \overline{2} \overline{1} . \overline{4} .58 . \overline{6} .7}_{\in W_{9}})=(1, \overline{3}, \overline{1}, 2, \overline{1}, 1) \|=9$.

The following proposition is easy to check (see Remark 2.11):

Proposition 2.18. If $w \in W_{n}$, then $\mathcal{U}_{n}^{\prime}(w)=\mathcal{A}_{\boldsymbol{C}(w)}$.

Remark. Mantaci and Reutenauer have defined the descent shape of a signed permutation [16]. It is a signed composition defined similarly than descent composition except that the absolute value of the letters in $u_{i}$ must be in increasing order. For instance, the descent shape of $9 . \overline{3} \cdot \overline{2} \cdot \overline{1} \overline{4} .58 . \overline{6} .7$ is $(1, \overline{1}, \overline{1}, \overline{2}, 2, \overline{1}, 1)$.

Example 2.19 - Let $n^{\prime}$ be a non-zero natural number, $n^{\prime}<n$ and let $c \in \mathbb{Z}$ such that $n-n^{\prime}=|c|$. Let $w \in W_{n^{\prime}} \subset W_{n}$ and write $\boldsymbol{C}(w)=\left(c_{1}, \ldots, c_{r}\right) \|=n^{\prime}$. Then $\boldsymbol{C}\left(\eta_{\left(n^{\prime}, c\right)} w\right)=\left(c_{1}, \ldots, c_{r}, c\right)$. Consequently, if $C \Vdash n$, an easy induction argument shows that $\boldsymbol{C}\left(\eta_{C}\right)=C$.

We have then defined a surjective map

$$
C: W_{n} \longrightarrow \operatorname{Comp}(n)
$$


whose fibers are equal to those of the application $\mathcal{U}_{n}^{\prime}: W_{n} \rightarrow \mathcal{P}\left(S_{n}^{\prime}\right)$. The surjectivity follows from Example 2.19 If $C \models n$, we define

$$
Y_{C}=\left\{w \in W_{n} \mid \boldsymbol{C}(w)=C\right\} .
$$

Then

$$
W_{n}=\coprod_{C \|=n} Y_{C}
$$

Example 2.20 - We have $Y_{n}=\left\{1_{n}\right\}, Y_{\bar{n}}=\left\{\sigma_{n} w_{n}\right\}, Y_{(1, \ldots, 1)}=\left\{\sigma_{n}\right\}$ and $Y_{(\overline{1}, \ldots, \overline{1})}=$ $\left\{w_{n}\right\}$.

First, note the following elementary facts.

Lemma 2.21. Let $C$ and $D$ be two signed compositions of $n$. Then:

(a) If $Y_{C} \cap X_{D} \neq \varnothing$, then $Y_{C} \subset X_{D}$.

(b) $\eta_{C} \in Y_{C}$ and $Y_{C} \subset X_{C}$.

Proof. (a) If $w \in W_{n}$, then $w \in X_{D}$ if and only if $\mathcal{U}_{n}^{\prime}(w)$ contains $S_{D}^{\prime}$. Since the map $w \mapsto \mathcal{U}_{n}^{\prime}(w)$ is constant on $Y_{C}$ (see Proposition 2.18), (a) follows.

(b) By Example 2.19] we have $\eta_{C} \in Y_{C} \cap X_{C}$. Therefore, by (a), $Y_{C} \subset X_{C}$.

We then define a relation $\leftarrow$ between signed composition of $n$ as follow. If $C$, $D \|=n$, we write $C \leftarrow D$ if $Y_{D} \subset X_{C}$. We denote by $\preccurlyeq$ the transitive closure of the relation $\leftarrow$. It follows from Lemma 2.21 (a) that

$$
X_{C}=\coprod_{C \leftarrow D} Y_{D}
$$

Example 2.23 - Let $w \in W_{n}$. By Remark 2.1 $w \in X_{\bar{n}}$ if and only if the sequence $(w(1), w(2), \ldots, w(n))$ of elements of $I_{n}$ is strictly increasing (see Remark 2.1). So there exists a unique $k \in\{0,1,2, \ldots, n\}$ such that $w(i)>0$ if and only if $i>k$. Note that $k=\ell_{t}(w)$. Let $i_{1}<\cdots<i_{k}$ be the sequence of elements of $I_{n}$ such that $(w(1), \ldots, w(k))=\left(\bar{i}_{k}, \ldots, \bar{i}_{1}\right)$. Then $w=r_{i_{1}} r_{i_{2}} \ldots r_{i_{k}}$ where, if $1 \leq i \leq n$, we set $r_{i}=s_{i-1} \ldots s_{2} s_{1} t$. Note that $\boldsymbol{C}(w)=(\bar{k}, n-k)$. Therefore,

$$
X_{\bar{n}}=\left\{r_{i_{1}} r_{i_{2}} \ldots r_{i_{k}} \mid 0 \leq k \leq n \text { and } 1 \leq i_{1}<i_{2}<\cdots<i_{k} \leq n\right\} .
$$

Note that $\ell\left(r_{i_{1}} r_{i_{2}} \ldots r_{i_{k}}\right)=i_{1}+i_{2}+\cdots+i_{k}$ and $\ell_{t}\left(r_{i_{1}} r_{i_{2}} \ldots r_{i_{k}}\right)=k$. We get

$$
X_{\bar{n}}=\coprod_{0 \leq k \leq n} Y_{(\bar{k}, n-k)},
$$

and, for every $k \in\{0,1,2, \ldots, n\}$, we have

$$
Y_{(\bar{k}, n-k)}=\left\{r_{i_{1}} r_{i_{2}} \ldots r_{i_{k}} \mid 1 \leq i_{1}<i_{2}<\cdots<i_{k} \leq n\right\} .
$$

This shows that $(\bar{n}) \leftarrow(\bar{k}, n-k)$.

Proposition 2.24. Let $C$ and $D$ be two signed compositions of $n$. Then:

(a) $C \leftarrow C$.

(b) If $C \subset D$, then $C \leftarrow D$.

(c) $\preccurlyeq$ is an order on $\operatorname{Comp}(n)$. 
Proof. (a) follows immediately from Lemma 2.21 (b).

(b) If $C \subset D$, then $X_{D} \subset X_{C}$. But, by Lemma 2.21 (b), we have $Y_{D} \subset X_{D}$. So $C \leftarrow D$.

(c) Let $a_{C}=\ell\left(\mu_{C}\right)$. By (a), $\preccurlyeq$ is reflexive. By definition, it is transitive. So it is sufficient to show that it is antisymmetric. But it follows from Lemma 2.21 (b) that:

- If $C \leftarrow D$, then $a_{D} \leq a_{C}$.

- If $C \leftarrow D$ and if $a_{C}=a_{D}$, then $C=D$.

The assertion (c) now follows easily from these two remarks.

Example 2.25 - If $C=\left(c_{1}, \ldots, c_{r}\right)$ is a composition of $n$ (not a signed composition), we will prove that

$$
\begin{aligned}
X_{\bar{n}}= & \coprod_{\substack{0 \leq m_{2} \leq c_{2} \\
0 \leq m_{3} \leq c_{3}}} X_{\left(\bar{c}_{1}, m_{2}, c_{2}-m_{2}, \ldots, m_{r}, c_{r}-m_{r}\right)}^{C} Y_{\left(\bar{c}_{1}, \bar{m}_{2}, c_{2}-m_{2}, \ldots, \bar{m}_{r}, c_{r}-m_{r}\right)}^{\left(\bar{c}_{1}, m_{2}, c_{2}-m_{2}, \ldots, m_{r}, c_{r}-m_{r}\right)} \sigma_{C, m_{2}, \ldots, m_{r}}^{-1}, \\
& \ldots \\
0 \leq & m_{r} \leq c_{r}
\end{aligned}
$$

where $\sigma_{C, m_{2}, \ldots, m_{r}} \in \mathfrak{S}_{n}$ satisfies

$$
\sigma_{C, m_{2}, \ldots, m_{r}}\left(S_{\left(c_{1}, m_{2}, c_{2}-m_{2}, \ldots, m_{r}, c_{r}-m_{r}\right)}^{\prime}\right) \subset S_{n}^{\prime}
$$

and

$$
\sigma_{C, m_{2}, \ldots, m_{r}} \in X_{\left(c_{1}, m_{2}, c_{2}-m_{2}, \ldots, m_{r}, c_{r}-m_{r}\right)} .
$$

By an easy induction argument, it is sufficient to prove it whenever $r=2$. In other words, we want to prove that, if $k+l=n$ with $k, l \geq 0$, then

$$
X_{\bar{n}}=\coprod_{0 \leq m \leq l} X_{(\bar{k}, m, l-m)}^{(\bar{k}, \bar{l})} Y_{(\bar{k}, \bar{m}, l-m)}^{(\bar{k}, m, l-m)} \sigma_{k, l, m}^{-1},
$$

where $\sigma_{k, l, m} \in \mathfrak{S}_{n}$ satisfies $\sigma_{k, l, m}\left(S_{k, m, l-m}^{\prime}\right) \subset S_{n}^{\prime}$ and $\sigma_{k, l, m} \in X_{(k, m, l-m)}$. But, if $0 \leq m \leq l$, we set

$$
\sigma_{k, l, m}(i)= \begin{cases}m+i & \text { if } 1 \leq i \leq k, \\ i-k & \text { if } k+1 \leq i \leq k+m, \\ i & \text { if } k+m+1 \leq i \leq n,\end{cases}
$$

and one can easily check that $(*)$ holds. Moreover, since $S_{k, m, l-m}^{\prime}=S_{n}^{\prime} \backslash\left\{s_{k}, s_{k+m}\right\}$, we get that $\sigma_{k, l, m}\left(S_{k, m, l-m}^{\prime}\right) \subset S_{n}^{\prime}$ and $\sigma_{k, l, m} \in X_{(k, m, l-m)}$.

\section{Generalized Descent algebra}

3.1. Definition. If $C$ and $D$ are two signed compositions of $n$ such that $C \subset D$, we set

and

$$
\begin{array}{ll}
x_{C}^{D}=\sum_{w \in X_{C}^{D}} w & \in \mathbb{Z} W_{D} \\
y_{C}^{D}=\sum_{w \in Y_{C}^{D}} w & \in \mathbb{Z} W_{D} .
\end{array}
$$

Now, let

$$
\Sigma^{\prime}\left(W_{D}\right)=\underset{C \subset D}{\oplus} \mathbb{Z} y_{C}^{D} \quad \subset \mathbb{Z} W_{D}
$$


Note that

$$
\Sigma^{\prime}\left(W_{D}\right)=\underset{C \subset D}{\oplus} \mathbb{Z} x_{C}^{D}
$$

by 2.22 and Proposition 2.24 We define

$$
\theta_{D}: \Sigma^{\prime}\left(W_{D}\right) \longrightarrow \mathbb{Z} \operatorname{Irr} W_{D}
$$

as the unique $\mathbb{Z}$-linear map such that

$$
\theta_{D}\left(x_{C}^{D}\right)=\operatorname{Ind}_{W_{C}}^{W_{D}} 1_{C}
$$

for every $C \subset D$. Here, $1_{C}$ is the trivial character of $W_{C}$. We denote by $\varepsilon_{D}$ the sign character of $W_{D}$.

Notation. If $D=(n)$, we set $x_{C}^{D}=x_{C}, y_{C}^{D}=y_{C}$ for simplification. If $E$ is $\mathbb{Z}$ module, we denote by $\mathbb{Q} E$ the $\mathbb{Q}$-vector space $\mathbb{Q} \otimes_{\mathbb{Z}} E$. We denote by $\theta_{D, \mathbb{Q}}$ the extension of $\theta_{D}$ to $\mathbb{Q} \Sigma^{\prime}\left(W_{D}\right)$ by $\mathbb{Q}$-linearity.

Remark. $\Sigma^{\prime}\left(W_{n}\right)$ contains the Solomon descent algebras of $W_{n}$ and $\mathfrak{S}_{n}$. Moreover, $\Sigma^{\prime}\left(W_{n}\right)$ is precisely the Mantaci-Reutenauer algebra which is, by definition, generated by $y_{D}=y_{D}^{(n)}$, for all $D \| n$.

3.2. First properties of $\boldsymbol{\theta}_{\boldsymbol{D}}$. By the Mackey formula for product of induced characters and by Proposition 2.13 we have that

$$
\theta_{n}\left(x_{C}\right) \theta_{n}\left(x_{D}\right)=\sum_{d \in X_{C D}} \theta_{n}\left(x_{d^{-1} C \cap D}\right) .
$$

Example 3.2 - If $C$ is parabolic or $D$ is semi-positive, then, by Corollary 2.17 we have

$$
x_{D}=\sum_{d \in X_{C D}} x_{C \cap \cap^{d} D}^{C} d .
$$

Therefore, by Proposition 2.8 (b) and Remark 2.15] we get

$$
x_{C} x_{D}=\sum_{d \in X_{C D}} x_{d^{-1} C \cap D} .
$$

So $x_{C} x_{D} \in \Sigma^{\prime}\left(W_{n}\right)$ and, by 3.1$] \theta_{n}\left(x_{C} x_{D}\right)=\theta_{n}\left(x_{C}\right) \theta_{n}\left(x_{D}\right)$.

Before starting the proof of the fact that $\Sigma^{\prime}\left(W_{D}\right)$ is a subalgebra of $\mathbb{Z} W_{D}$ and that $\theta_{D}$ is a morphism of algebras, we need the following result, which will be useful for arguing by induction. If $C \subset D$, the transitivity of induction and Proposition 2.8 (b) show that the diagram

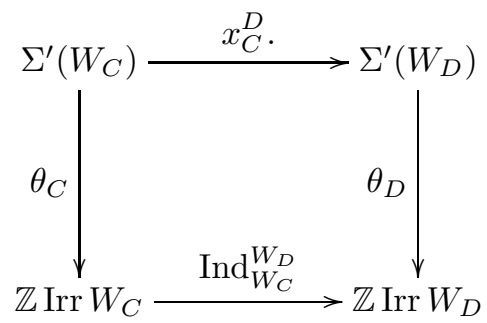

is commutative.

Now, let $p_{D}: W_{D} \rightarrow \mathfrak{S}_{D^{+}}$be the canonical projection. It induces an injective morphism of $\mathbb{Z}$-algebras $p_{D}^{*}: \mathbb{Z} \operatorname{Irr} \mathfrak{S}_{D^{+}} \rightarrow \mathbb{Z} \operatorname{Irr} W_{D}$. Moreover, the algebra 
$\Sigma^{\prime}\left(\mathfrak{S}_{D^{+}}\right)$coincides with the usual descent algebra in symmetric groups and is contained in $\Sigma^{\prime}\left(W_{D}\right)$. Also, the diagram

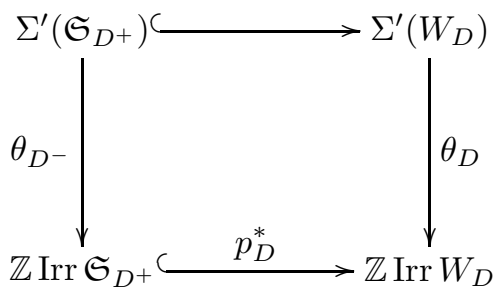

is commutative.

Example 3.5 - We have $y_{(\overline{1}, \ldots, \overline{1})}=w_{n}, y_{\bar{n}}=w_{n} \sigma_{n}=\sigma_{n} w_{n}, y_{n}=1$ and $y_{(1, \ldots, 1)}=$ $\sigma_{n}$. It is well-known [18 that $y_{(\overline{1}, \ldots, \overline{1})}$ belongs to the classical descent algebra of $W_{n}$ and that

$$
\theta_{n}\left(w_{n}\right)=\varepsilon_{n} .
$$

On the other hand,

$$
\theta_{n}\left(1_{n}\right)=1_{(n)} .
$$

Also, by the commutativity of the diagram 3.4 and as above, we have

$$
\theta_{n}\left(\sigma_{n}\right)=\gamma_{n}
$$

where $\gamma_{n}=p_{n}^{*} \varepsilon_{\bar{n}}$. Finally, $w_{n}$ is a $\mathbb{Z}$-linear combination of $x_{C}$, where $C$ runs over the parabolic compositions of $n$. Therefore, by Example 3.2 we have, for every $x \in \Sigma^{\prime}\left(W_{n}\right)$,

$$
\theta_{n}\left(w_{n} x\right)=\theta_{n}\left(w_{n}\right) \theta_{n}(x)=\varepsilon_{n} \theta_{n}(x) .
$$

In particular,

$$
\theta_{n}\left(y_{\bar{n}}\right)=\theta_{n}\left(w_{n} \sigma_{n}\right)=\varepsilon_{n} \gamma_{n} .
$$

So we have obtained the four linear characters of $W_{n}$ as images by $\theta_{n}$ of explicit elements of $\Sigma^{\prime}\left(W_{n}\right)$.

Let $\operatorname{deg}_{D}: \mathbb{Z} \operatorname{Irr} W_{D} \rightarrow \mathbb{Z}$ be the $\mathbb{Z}$-linear map sending an irreducible character of $W_{D}$ to its degree. It is a morphism of $\mathbb{Z}$-algebras. Let $\operatorname{aug}_{D}: \mathbb{Z} W_{D} \rightarrow \mathbb{Z}$ be the augmentation morphism, then it is clear that the diagram

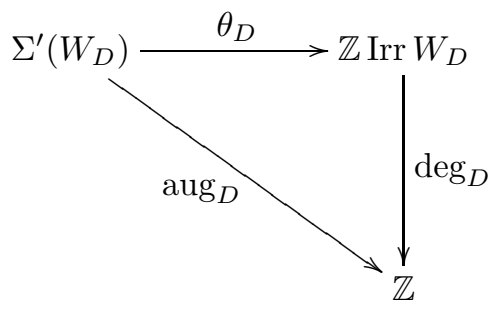

is commutative. 
3.3. Main result. We are now ready to prove that $\Sigma^{\prime}\left(W_{D}\right)$ is a $\mathbb{Z}$-subalgebra of $\mathbb{Z} W_{D}$ and that $\theta_{D}$ is a surjective morphism of algebras.

Theorem 3.7. Let $D$ be a signed composition of $n$, then:

(a) $\Sigma^{\prime}\left(W_{D}\right)$ is a $\mathbb{Z}$-subalgebra of $\mathbb{Z} W_{D}$;

(b) $\theta_{D}$ is a morphism of algebra;

(c) $\theta_{D}$ is surjective and $\operatorname{Ker} \theta_{D}=\bigoplus_{C, C^{\prime} \subset D} \mathbb{Z}\left(x_{C}^{D}-x_{C^{\prime}}^{D}\right)$;

(d) $\operatorname{Ker} \theta_{D, \mathbb{Q}}$ is the radical of the algebra $\mathbb{Q} \Sigma^{\prime}\left(W_{D}\right)$. Moreover, $\mathbb{Q} \Sigma^{\prime}\left(W_{D}\right)$ is a split algebra whose largest semisimple quotient is commutative. In particular, all its simple modules are of dimension 1.

Proof. We want to prove the theorem by induction on $\left|W_{D}\right|$. By taking direct products, we may therefore assume that $D=(n)$ or $D=(\bar{n})$. If $D=(\bar{n})$, then it is well-known that (a), (b), (c) and (d) hold. So we may assume that $D=(n)$ and that (a), (b), (c) and (d) hold for every signed composition $D^{\prime}$ of $n$ different from $(n)$.

(a) and (b): Let $A$ and $B$ be two signed compositions of $n$. We want to prove that $x_{A} x_{B} \in \Sigma^{\prime}\left(W_{n}\right)$ and that $\theta_{n}\left(x_{A} x_{B}\right)=\theta_{n}\left(x_{A}\right) \theta_{n}\left(x_{B}\right)$. If $A$ is parabolic or $B$ is semi-positive, then this is just Example 3.2 So we may assume that $A$ is not parabolic and $B$ is not semi-positive.

First, note that $B \subset B^{+}$and that $B^{+}$is semi-positive. Therefore, by Proposition 2.8 (b) and Example 3.2 we have

$$
x_{A} x_{B}=x_{A} x_{B^{+}} x_{B}^{B^{+}}=\sum_{d \in X_{A, B^{+}}} x_{d^{-1} A \cap B^{+}} x_{B}^{B^{+}}=x_{B^{+}} \sum_{d \in X_{A, B^{+}}} x_{d^{-1} A \cap B^{+}}^{B^{+}} x_{B}^{B^{+}} .
$$

Assume first that $B^{+} \neq(n)$. Then, by induction hypothesis,

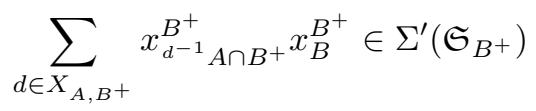

and

$$
\theta_{B^{+}}\left(\sum_{d \in X_{A, B^{+}}} x_{d^{-1} A \cap B^{+}}^{B^{+}} x_{B}^{B^{+}}\right)=\sum_{d \in X_{A, B^{+}}} \theta_{B^{+}}\left(x_{d^{-1}}^{B^{+}} A \cap B^{+}\right) \theta_{B^{+}}\left(x_{B}^{B^{+}}\right) .
$$

Therefore, by 3.3 and $3.4 x_{A} x_{B} \in x_{B^{+}} \Sigma^{\prime}\left(\mathfrak{S}_{B^{+}}\right) \subset \Sigma^{\prime}\left(\mathfrak{S}_{n}\right) \subset \Sigma^{\prime}\left(W_{n}\right)$ and, by 3.3 and by the Mackey formula for tensor product, we get

$$
\begin{aligned}
\theta_{n}\left(x_{A} x_{B}\right) & =\operatorname{Ind}_{W_{B^{+}}}^{W_{n}}\left(\sum _ { d \in X _ { A , B ^ { + } } } \theta _ { B ^ { + } } \left(x_{d^{-1}}^{B^{+}} A \cap B^{+}\right.\right. \\
& \left.\theta_{B^{+}}\left(x_{B}^{B^{+}}\right)\right) \\
& =\theta_{n}\left(x_{A}\right) \operatorname{Ind}_{W_{B} W_{n}}^{W_{n}} \theta_{B^{+}}\left(x_{B}^{B^{+}}\right) \\
& =\theta_{n}\left(x_{A}\right) \theta_{n}\left(x_{B}\right),
\end{aligned}
$$

as desired.

Therefore, it remains to consider the case where $B^{+}=(n)$. In particular, $B=$ $(n)$ or $(\bar{n})$. Since $B$ is not semi-positive, we have $B=(\bar{n})$. By Example 2.25 we have

$$
x_{\bar{n}}=x_{A^{-}}^{A^{+}}+\sum_{D \subset A^{+}} a_{D} x_{D}^{A^{+}}\left(\sigma_{D}^{-1}-1\right)
$$


where $a_{D} \in \mathbb{Z}$ and $\sigma_{D}\left(S_{D}^{\prime}\right) \subset S_{n}^{\prime}$ and $\sigma_{D} \in X_{D}$ for every $D \subset A^{+}$. Therefore,

$$
x_{A} x_{B}=x_{A} x_{\bar{n}}=x_{A^{+}}\left(x_{A}^{A^{+}} x_{A^{-}}^{A^{+}}+\sum_{D \subset A^{+}} a_{D} x_{A}^{A^{+}} x_{D}^{A^{+}}\left(\sigma_{D}^{-1}-1\right)\right) .
$$

Now, $W_{A}$ is a standard parabolic subgroup of $W_{A^{+}}$. So, by Example 3.2 we have

$$
x_{A} x_{B}=\sum_{d \in X_{A, A^{-}}^{A^{+}}} x_{d^{-1} A \cap\left(A^{-}\right)}+\sum_{D \subset A^{+}}\left(a_{D} \sum_{d \in X_{A, D}^{A+}} x_{d^{-1} A \cap D}\left(\sigma_{D}^{-1}-1\right)\right) .
$$

Therefore, since $\sigma_{D}\left(S_{D}^{\prime}\right) \subset S_{n}^{\prime}$ and $\sigma_{D} \in X_{D}$, we have that $x_{d^{-1} A \cap D} \sigma_{D}^{-1}=$ $x_{\sigma_{D}\left(d^{-1} A \cap D\right)}$. So $x_{A} x_{B} \in \Sigma^{\prime}\left(W_{n}\right)$ and $\theta_{n}\left(x_{A} x_{B}\right)=\theta_{n}\left(x_{A}\right) \theta_{n}\left(x_{B}\right)$ by the Mackey formula for tensor product of induced characters. This concludes the proof of (a) and (b). Indeed, the surjectivity of $\theta_{n}$ is well-known.

(c) First, let us show that $\theta_{n}$ is surjective. Using the induction hypothesis, the commutativity of the diagram 3.3 and the classical description of irreducible characters of $W_{n}$, we are reduced to prove that, for every $\chi \in \operatorname{Irr} \mathfrak{S}_{n}, p_{n}^{*}(\chi)$ and $p_{n}^{*}(\chi) \varepsilon_{n}$ lie in the image of $\theta_{n}$. But it is well-known that $\theta_{\bar{n}}$ is surjective. So the result follows from the commutativity of the diagram 3.4 and from Example 3.5 (d).

Now, let $I=\sum_{C, C^{\prime} \|=n} \mathbb{Z}\left(x_{C}-x_{C^{\prime}}\right)$. Then it is clear that $I \subset \operatorname{Ker} \theta_{n}$. Let

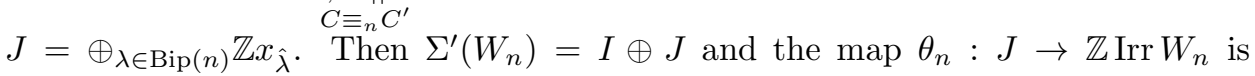
surjective. Since $J$ and $\mathbb{Z} \operatorname{Irr} W_{n}$ have the same rank (equal to $|\operatorname{Bip}(n)|$ ), we get that $J \cap \operatorname{Ker} \theta_{n}=0$. So $I=\operatorname{Ker} \theta_{n}$.

(d) Let $R=\operatorname{Rad}\left(\mathbb{Q} \Sigma^{\prime}\left(W_{n}\right)\right)$ and $K=\operatorname{Ker} \theta_{n, \mathbb{Q}}$. Since $\operatorname{Im}\left(\theta_{n, \mathbb{Q}}\right)=\mathbb{Q} \operatorname{Irr} W_{n}$ is a semisimple algebra, we get that $R \subset K$.

Now, let $\chi: \mathbb{Q} W_{n} \rightarrow \mathbb{Q}$ be the character of the $\mathbb{Q} W_{n}$-module $\mathbb{Q} W_{n}$ (the regular representation). Then, $\chi(w)=0$ for every $w \neq 1$. Let $\chi^{\prime}$ denote the restriction of $\chi$ to $\Sigma^{\prime}\left(W_{n}\right)$. We have $\chi^{\prime}\left(x_{C}\right)=\chi(1)$ for every $C \| n$. Therefore, $\chi^{\prime}(x)=0$ for every $x \in K$ by (c). We fix now $x \in K$. Then, for every $y \in \mathbb{Q} \Sigma^{\prime}\left(W_{n}\right)$, we have $\chi^{\prime}(x y)=0$ because $x y \in K$ by (b). Since the $\mathbb{Q} \Sigma^{\prime}\left(W_{n}\right)$-module $\mathbb{Q} W_{n}$ is faithful, this implies that $x \in R$. So $K \subset R$.

Remark. $\Sigma^{\prime}\left(W_{C}\right) \simeq \Sigma^{\prime}\left(W_{c_{1}}\right) \otimes \Sigma^{\prime}\left(W_{c_{2}}\right) \otimes \cdots \otimes \Sigma^{\prime}\left(W_{c_{r}}\right)$.

3.4. Further properties of $\boldsymbol{\theta}_{\boldsymbol{D}}$. Let $\tau_{D}: \mathbb{Z} W_{D} \rightarrow \mathbb{Z}$ be the unique linear map such that $\tau_{D}(w)=0$ if $w \neq 1$ and $\tau_{D}(1)=1$. Then $\tau_{D}$ is the canonical symmetrizing form on $\mathbb{Z} W_{D}$ : in particular, the map $\mathbb{Z} W_{D} \times \mathbb{Z} W_{D} \rightarrow \mathbb{Z},(x, y) \mapsto \tau_{D}(x y)$ is a nondegenerate symmetric bilinear form on $\mathbb{Z} W_{D}$. We denote by $\langle., .\rangle_{D}$ the scalar product on $\mathbb{Z} \operatorname{Irr} W_{D}$ such that $\operatorname{Irr} W_{D}$ is an orthonormal basis. The following property is a kind of "isometry property" for the morphism $\theta_{D}$.

Proposition 3.8. If $x, y \in \Sigma^{\prime}\left(W_{D}\right)$, then $\tau_{D}(x y)=\left\langle\theta_{D}(x), \theta_{D}(y)\right\rangle_{D}$.

Proof. Let $C$ and $C^{\prime}$ be two signed compositions of $n$ such that $C, C^{\prime} \subset D$. Then $\tau_{D}\left(x_{C}^{D} x_{C^{\prime}}^{D}\right)=\left|X_{C C^{\prime}}^{D}\right|$ by definition of $\tau_{D}$. Moreover, since $\theta_{D}\left(x_{C}\right)$ and $\theta_{D}\left(x_{C^{\prime}}\right)$ take only rational values, we have

$$
\left\langle\theta_{D}\left(x_{C}^{D}\right), \theta_{D}\left(x_{C^{\prime}}^{D}\right)\right\rangle_{D}=\left\langle\theta_{D}\left(x_{C}^{D}\right) \theta_{D}\left(x_{C^{\prime}}^{D}\right), 1_{W_{D}}\right\rangle_{D} .
$$


But, by [3.1 and by Frobenius reciprocity, we have

$$
\left\langle\theta_{D}\left(x_{C}^{D}\right) \theta_{D}\left(x_{C^{\prime}}^{D}\right), 1_{W_{D}}\right\rangle_{D}=\left|X_{C C^{\prime}}^{D}\right| .
$$

So the proposition follows now from the fact that $\left(x_{C}^{D}\right)_{C \subset D}$ generates $\Sigma^{\prime}\left(W_{D}\right)$.

Corollary 3.9. $\operatorname{Ker} \theta_{D}=\left\{x \in \Sigma^{\prime}\left(W_{D}\right) \mid \forall y \in \Sigma^{\prime}\left(W_{D}\right), \tau_{D}(x y)=0\right\}$.

Proof. Since $\langle., .\rangle_{D}$ is non-degenerate on $\mathbb{Z} \operatorname{Irr} W_{D}$, this follows from Proposition 3.8

Write $D=\left(d_{1}, \ldots, d_{r}\right)$ and let $\operatorname{Bip}(D)$ denote the set of $r$-uples $\left(\lambda_{(1)}, \ldots, \lambda_{(r)}\right)$ of bipartitions $\lambda_{(i)}=\left(\lambda_{(i)}^{+}, \lambda_{(i)}^{-}\right)$such that $\lambda_{(i)}^{-}=\emptyset$ if $d_{i}<0$ and $\left|\lambda_{(i)}\right|=\left|d_{i}\right|$ for every $i \in[1, r]$. If $\lambda \in \operatorname{Bip}(D)$, we denote by $\mathcal{C}_{\lambda}^{D}$ the conjugacy class in $W_{D}$ of a Coxeter element of $W_{\hat{\lambda}}$ (with respect to $S_{\hat{\lambda}}$ ). Let $f_{\lambda}^{D}$ denote the characteristic function of $\mathcal{C}_{\lambda}^{D}$. Then $f_{\lambda}$ is a primitive idempotent of $\mathbb{Q} \operatorname{Irr} W_{n}$. Moreover, $\left(f_{\lambda}^{D}\right)_{\lambda \in \operatorname{Bip}(D)}$ is a complete family of orthogonal primitive idempotents of $\mathbb{Q} \operatorname{Irr} W_{D}$. Since $\theta_{D}$ is surjective, there exists a family of idempotents $\left(E_{\lambda}^{D}\right)_{\lambda \in \operatorname{Bip}(D)}$ of $\mathbb{Q} \Sigma^{\prime}\left(W_{D}\right)$ such that

(1) $\forall \lambda \in \operatorname{Bip}(D), \theta_{D}\left(E_{\lambda}^{D}\right)=f_{\lambda}^{D}$.

(2) $\forall \lambda, \mu \in \operatorname{Bip}(D), \lambda \neq \mu \Rightarrow E_{\lambda}^{D} E_{\mu}^{D}=E_{\mu}^{D} E_{\lambda}^{D}=0$.

(3) $\sum_{\lambda \in \operatorname{Bip}(D)} E_{\lambda}^{D}=1$.

Proposition 3.10. If $x \in \Sigma^{\prime}\left(W_{D}\right)$, then

$$
\theta_{D}(x)=\left|W_{D}\right| \sum_{\lambda \in \Vdash D} \frac{\tau_{D}\left(x E_{\lambda}^{D}\right)}{\left|\mathcal{C}_{\lambda}^{D}\right|} f_{\lambda}^{D} \in \mathbb{Z} \operatorname{Irr} W_{D} .
$$

Proof. If $f \in \mathbb{Q} \operatorname{Irr} W_{D}$, then

$$
f=\left|W_{D}\right| \sum_{\lambda \in \operatorname{Bip}(D)} \frac{\left\langle f, f_{\lambda}^{D}\right\rangle_{D}}{\left|\mathcal{C}_{\lambda}^{D}\right|} f_{\lambda}^{D} \in \mathbb{Z} \operatorname{Irr} W_{D} .
$$

If $f=\theta_{D}(x)$ with $x \in \Sigma^{\prime}\left(W_{D}\right)$, then we get the desired formula just by applying Proposition 3.8 and the property (1) above.

3.5. Character table. Since all irreducible characters of $W_{D}$ have rational values, the algebra $\mathbb{Q} \operatorname{Irr} W_{D}$ may be identified with the $\mathbb{Q}$-algebra of central functions $W_{D} \rightarrow \mathbb{Q}$. If $\lambda \in \operatorname{Bip}(D)$, we denote by $\operatorname{ev}_{\lambda}^{D}: \mathbb{Q} \operatorname{Irr} W_{D} \rightarrow \mathbb{Q}, \chi \mapsto \chi\left(c_{\lambda}^{D}\right)$, where $c_{\lambda}^{D}$ is some element of $\mathcal{C}_{\lambda}^{D}$ (for instance, a Coxeter element of $W_{\hat{\lambda}}$ ). Then $\operatorname{ev}_{\lambda}^{D}$ is a morphism of algebras: it is an irreducible representation of $\mathbb{Q} \operatorname{Irr} W_{D}$. Moreover, $\left\{\operatorname{ev}_{\lambda}^{D} \mid \lambda \in \operatorname{Bip}(D)\right\}$ is a complete set of representatives of isomorphy classes of irreducible representations of $\mathbb{Q} \operatorname{Irr} W_{D}$. Now, let $\mathbb{Q}_{\lambda}^{D}$ denote the $\mathbb{Q} \Sigma^{\prime}\left(W_{D}\right)$-module whose underlying vector space is $\mathbb{Q}$ and on which an element $x \in \mathbb{Q} \Sigma^{\prime}\left(W_{D}\right)$ acts by multiplication by $\pi_{\lambda}^{D}(x)=\left(\operatorname{ev}_{\lambda}^{D} \circ \theta_{D}\right)(x)$. Then, by Theorem 3.7 we get:

Proposition 3.11. $\left\{\mathbb{Q}_{\lambda}^{D} \mid \lambda \in \operatorname{Bip}(D)\right\}$ is a complete set of isomorphy classes of $\mathbb{Q} \Sigma^{\prime}\left(W_{D}\right)$-modules. We have

$$
\operatorname{Irr}\left(\mathbb{Q} \Sigma^{\prime}\left(W_{D}\right)\right)=\left\{\pi_{\lambda}^{D} \mid \lambda \in \operatorname{Bip}(D)\right\} .
$$


The character table of $\mathbb{Q} \Sigma^{\prime}\left(W_{D}\right)$ is the square matrix whose rows and the columns are indexed by $\operatorname{Bip}(D)$ and whose $(\lambda, \mu)$-entry is the value of the irreducible character $\pi_{\lambda}^{D}\left(x_{\hat{\mu}}^{D}\right)$. Note that

$$
\pi_{\lambda}^{D}\left(x_{\hat{\mu}}^{D}\right)=\left(\operatorname{Ind}_{W_{\hat{\mu}}}^{W_{D}} 1_{\hat{\mu}}\right)\left(c_{\lambda}^{D}\right)
$$

Notation. If $D=(n)$, we denote $\mathcal{C}_{\lambda}^{D}, f_{\lambda}^{D}, E_{\lambda}^{D}, c_{\lambda}^{D}, \operatorname{ev}_{\lambda}^{D}, \mathbb{Q}_{\lambda}^{D}$ and $\pi_{\lambda}^{D}$ by $\mathcal{C}_{\lambda}, f_{\lambda}, E_{\lambda}$, $c_{\lambda}, \mathrm{ev}_{\lambda}, \mathbb{Q}_{\lambda}$ and $\pi_{\lambda}$ respectively.

Now, if $\lambda, \mu \in \operatorname{Bip}(D)$, we write $\lambda \subset \mu$ if there exists some $w \in W_{D}$ such that $W_{\hat{\lambda}} \subset{ }^{w} W_{\hat{\mu}}$. By Proposition 2.7 $\subset$ is a partial order on $\operatorname{Bip}(D)$. For this partial order, the character table of $\mathbb{Q} \Sigma^{\prime}\left(W_{D}\right)$ is triangular :

Proposition 3.12. If $\pi_{\lambda}^{D}\left(x_{\hat{\mu}}^{D}\right) \neq 0$, then $\lambda \subset \mu$.

Proof. We may, and we will, assume that $D=(n)$. If $\pi_{\lambda}\left(x_{\hat{\mu}}\right) \neq 0$, then there exists $w \in W_{n}$ such that $w c_{\lambda} w^{-1} \in W_{\hat{\mu}}$. Therefore, there exists $\nu \in \operatorname{Bip}(\hat{\mu})$ and $w^{\prime} \in W_{\hat{\mu}}$ such that $w^{\prime} w c_{\lambda} w^{-1} w^{\prime-1}$ is a Coxeter element of $W_{\hat{\nu}}$. Let $C$ denote the unique signed composition of $n$ such that $W_{\hat{\nu}}=W_{C}$ and let $\lambda^{\prime}=\lambda(C)$. Then $w^{\prime} w c_{\lambda} w^{-1} w^{\prime-1}$ is conjugate to $c_{\lambda^{\prime}}$. Therefore, $\lambda=\lambda^{\prime}$. This completes the proof of the proposition.

In the last section of this paper, we will give the character table of $\Sigma^{\prime}\left(W_{2}\right)$.

3.6. Combinatorial description. In $\mathfrak{S}_{n}$, the refinement of compositions is useful to construct $X_{C}$ from $Y_{D}$ without considering subsets of $S_{\bar{n}}^{\prime}$. The aim of this part is to describe such a procedure in our case. Start with an example, consider $C=(\overline{2}, 1)$, then the subsets of $S_{3}^{\prime}$ containing $S_{C}=\left\{s_{1}, t_{3}\right\}$ are $\left\{s_{1}, s_{2}, t_{3}\right\}=\mathcal{A}_{(\overline{2}, 1)}$; $\left\{s_{1}, t_{2}, t_{3}\right\}=\mathcal{A}_{(\overline{1}, 1,1)} ;\left\{s_{1}, t_{1}, t_{2}, t_{3}\right\}=\mathcal{A}_{(2,1)},\left\{s_{1}, s_{2}, t_{2}, t_{3}\right\}=\mathcal{A}_{(\overline{1}, 2)}$ and $S_{3}^{\prime}=\mathcal{A}_{(3)}$. Observe that $(1,2)$ (which corresponds to $\left\{s_{2}, t_{1}, t_{2}, t_{3}\right\} \not \supset S_{C}$ ) is not obtained. Here, we define a procedure which give $(\overline{2}, 1),(\overline{1}, 1,1),(\overline{1}, 2)$ and $(3)$, without to obtain $(1,2)$.

Let $C=\left(c_{1}, \ldots, c_{k}\right) \| n$, we write:

- $C \stackrel{B}{\longleftarrow} D$ if $D=\left(a_{1}, b_{1}, a_{2}, b_{2}, \ldots, a_{k}, b_{k}\right) \| n$ such that for all $i \in[1, k]$ we have $\left|a_{i}\right|+\left|b_{i}\right|=\left|c_{i}\right| ; a_{i}=c_{i}$ (hence $b_{i}=0$ ) if $c_{i}>0 ; a_{i} \leq 0 \leq b_{i}$ if $c_{i}<0$ (remove the 0 from the list $\left(a_{1}, b_{1}, a_{2}, b_{2}, \ldots, a_{k}, b_{k}\right)$ ). That is, $D$ is obtained from $C$ by broken negative parts operations.

- $C \stackrel{R}{\longleftarrow} D$ if $C$ is finer that $D \| n$, that is, $D$ can be obtained from $C$ by summing consecutive parts of $C$ having the same sign (refinement operations).

Example 3.13 - Let $C=(1, \overline{2}, \overline{1})$, then

$$
\{D \| 4 \mid C \stackrel{B}{\longleftarrow} D\}=\{(1, \overline{2}, \overline{1}),(1, \overline{1}, 1, \overline{1}),(1, \overline{1}, 1,1),(1,2, \overline{1}),(1, \overline{2}, 1),(1,2,1)\} .
$$

Remark 3.14 - Let $C, D \|=n$, then we have $C \leftarrow D$ if and only if $S_{C} \subset \mathcal{A}_{D}$. We deduce easily from definitions, Lemma 2.21 and Example 2.23 the following properties for any $i \in[1, k-1]$ :

- if $\operatorname{sign} c_{i}=\operatorname{sign} c_{i+1}, C=\left(c_{1}, \ldots, c_{i}, c_{i+1}, \ldots, c_{k}\right) \stackrel{R}{\longleftarrow}\left(c_{1}, \ldots, c_{i}+c_{i+1}, \ldots, c_{k}\right)=$ $D$, and this means that $\mathcal{A}_{D}=\mathcal{A}_{C} \uplus\left\{s_{\left|c_{1}\right|+\cdots+\left|c_{i}\right|}\right\}$; 
- if $c_{i}, c_{i+1}<0$, then

$$
C=\left(c_{1}, \ldots, c_{i}, c_{i+1}, \ldots, c_{k}\right) \stackrel{B}{\longleftarrow}\left(c_{1}, \ldots, c_{i}+1,1, c_{i+1}, \ldots, c_{k}\right)=D
$$

(remove the 0 from the list), and this means that $\mathcal{A}_{D}=\mathcal{A}_{C} \uplus\left\{t_{\left|c_{1}\right|+\cdots+\left|c_{i}\right|}\right\}$;

- if $c_{i}<0$ and $c_{i+1}>0$, then

$$
C=\left(c_{1}, \ldots, c_{i}, c_{i+1}, \ldots, c_{k}\right) \stackrel{B}{\longleftarrow}\left(c_{1}, \ldots, c_{i}+1,1, c_{i+1}, \ldots, c_{k}\right)=D
$$

(remove the 0 from the list), and this means that $\mathcal{A}_{D} \uplus\left\{s_{\left|c_{1}\right|+\cdots+\left|c_{i}\right|}\right\}=$ $\mathcal{A}_{C} \uplus\left\{t_{\left|c_{1}\right|+\cdots+\left|c_{i}\right|}\right\}$. Moreover, as $s_{\left|c_{1}\right|+\cdots+\left|c_{i}\right|} \notin S_{C}$, we have $S_{C} \subset \mathcal{A}_{D}$, that is, $C \leftarrow D$;

- finally, if $c_{i}<0$, then $C \stackrel{B}{\longleftarrow}\left(c_{1}, \ldots, c_{i}+1,1, c_{i+1}, \ldots, c_{k}\right)=D$ and

$$
C \stackrel{B}{\longleftarrow}\left(c_{1}, \ldots, c_{i}+2,2, c_{i+1}, \ldots, c_{k}\right)=D^{\prime}
$$

(remove the 0 from the list), and this means that $\mathcal{A}_{D^{\prime}}=\mathcal{A}_{D} \uplus\left\{t_{\left|c_{1}\right|+\cdots+\left|c_{i}\right|}\right\}$. Hence $S_{C} \subset \mathcal{A}_{D} \subset \mathcal{A}_{D^{\prime}}$.

In all these cases, we have $C \leftarrow D$.

Theorem 3.15. Let $C, D \Vdash n$, then $C \leftarrow D$ if and only if there is $E \| n$ such that $C \stackrel{B}{\longleftarrow} \stackrel{R}{\longleftarrow} D$. Moreover, $E$ is uniquely determined.

Proof. Suppose that $E$ exists, then it is easy to check (using Remark 3.14 and induction) that $S_{C} \subset \mathcal{A}_{E} \subset \mathcal{A}_{D}$, which implies $C \leftarrow D$.

Now, suppose that $C \leftarrow D$. As $S_{C} \subset \mathcal{A}_{D}$, it is easy to construct a unique $E \|=n$ such that $\mathcal{A}_{E} \cap T_{n}=\mathcal{A}_{D} \cap T_{n}$ and $C \stackrel{B}{\longleftarrow} E$ (hence $C \leftarrow B$ ). It remains to show that $E \stackrel{R}{\longleftarrow} D$, that is, to show that $\mathcal{A}_{E} \cap S_{\bar{n}} \subset \mathcal{A}_{D} \cap S_{\bar{n}}$. Let $s_{j} \in \mathcal{A}_{E} \cap S_{\bar{n}}$, then either $j \in\left[\left|c_{1}\right|+\cdots+\left|c_{i-1}\right|+1, \ldots,\left|c_{1}\right|+\cdots+\left|c_{i}\right|-1\right]$ hence $s_{j} \in S_{C} \subset \mathcal{A}_{D}$; or $j=\left|c_{1}\right|+\cdots+\left|c_{i}\right|$ and $c_{i}<0$ and $c_{i+1}>0$ by definition. But refinement operations do not act on parts having not the same sign, that is, $s_{j} \in \mathcal{A}_{D}$.

Example 3.16 - Consider the signed composition $C=(1, \overline{2}, \overline{1})$. Then we obtain from Theorem 3.15 and Example 3.13

$$
\begin{aligned}
& X_{(1, \overline{2}, \overline{1})}=Y_{(1, \overline{2}, \overline{1})} \cup Y_{(1, \overline{3})} \cup Y_{(1, \overline{1}, 1, \overline{1})} \cup Y_{(1, \overline{1}, 1,1)} \cup Y_{(1, \overline{1}, 2)} \cup Y_{(1,2, \overline{1})} \cup Y_{(3, \overline{1})} \\
& \cup Y_{(1, \overline{2}, 1)} \cup Y_{(1,2,1)} \cup Y_{(3,1)} \cup Y_{(1,3)} \cup Y_{(4)} \text {. }
\end{aligned}
$$

\section{Coplactic space}

4.1. Robinson-Schensted correspondence for $\boldsymbol{W}_{\boldsymbol{D}}$. In [20, the author defined a bijection between $W_{n}$ and a certain set of bitableaux, which sounds like a Robinson-Schensted correspondence. Let us recall here some of his results. A bitableau is a pair $T=\left(T^{+}, T^{-}\right)$of tableaux. The shape of $T$ is the bipartition $\left(\lambda^{+}, \lambda^{-}\right)$, where $\lambda^{+}$is the shape of $T^{+}$and $\lambda^{-}$is the shape of $T^{-}$: it is denoted by $\operatorname{sh} T$. We note $|T|=|\operatorname{sh} T|$. The bitableau $T$ is said to be standard if the set of numbers in $T^{+}$and $T^{-}$is $[1, m]$, where $m=|T|$, and if the fillings of $T^{+}$and $T^{-}$ are increasing in rows and in column.

Let $D \| n$. Write $D=\left(d_{1}, \ldots, d_{r}\right)$ and denote by $\mathcal{S B T}(D)$ the set of $r$-uples $T=\left(T_{1}, \ldots, T_{r}\right)$ of bitableaux $T_{i}=\left(T_{i}^{+}, T_{i}^{-}\right)$such that $\left|T_{i}\right|=\left|d_{i}\right|, T_{i}^{-}=\emptyset$ if $d_{i}<0, T_{i}^{+}$and $T_{i}^{-}$are standard and the fillings of $T_{i}^{+}$and $T_{i}^{-}$are exactly the numbers in $I_{D,+}^{(i)}=\left[\left|d_{1}\right|+\cdots+\left|d_{i-1}\right|+1,\left|d_{1}\right|+\cdots+\left|d_{i}\right|\right]$. The shape of $T$, 
denoted by $\operatorname{sh} T$, is the $r$-uple of bipartitions $\left(\operatorname{sh} T_{1}, \ldots, \operatorname{sh} T_{r}\right)$. If $T \in \mathcal{S B T}(D)$, then $\operatorname{sh} T \in \operatorname{Bip}(D)$. If $\lambda \in \operatorname{Bip}(D)$, we denote by $\mathcal{S B T}_{\lambda}^{D}$ the set of elements $T \in \mathcal{S B T}(D)$ such that $\operatorname{sh} T=\lambda$. In $[20$, the author defined a bijection (which we call generalized Robinson-Schensted correspondence)

$$
\begin{aligned}
\pi_{D}: W_{D} & \longrightarrow\{(P, Q) \in \mathcal{S B T}(D) \times \mathcal{S B T}(D) \mid \operatorname{sh} P=\operatorname{sh} Q\} \\
w & \longmapsto\left(\boldsymbol{P}_{D}(w), \boldsymbol{Q}_{D}(w)\right) .
\end{aligned}
$$

Note that, in 20 (see also [5 Section 3]), the bijection has been defined only for $D=(n)$. It is not difficult to deduce from this the bijection $\pi_{D}$ for general $D$. To this bijection is associated a partition of $W_{n}$ as follows: if $Q \in \mathcal{S B T}(D)$, we set

$$
Z_{Q}^{D}=\left\{w \in W_{D} \mid \boldsymbol{Q}_{D}(w)=Q\right\} .
$$

Then

$$
W_{D}=\coprod_{Q \in \mathcal{S B \mathcal { T }}(D)} Z_{Q}^{D}
$$

4.2. Properties. First, note that the bijection $\pi_{D}$ satisfies the following property: if $w \in W_{n}$, then

$$
\pi_{D}\left(w^{-1}\right)=\left(\boldsymbol{Q}_{D}(w), \boldsymbol{P}_{D}(w)\right) .
$$

In particular, if $Q$ and $Q^{\prime}$ are two elements of $\mathcal{S B T}(D)$, then

$$
\left|Z_{Q}^{D} \cap\left(Z_{Q^{\prime}}^{D}\right)^{-1}\right|= \begin{cases}1 & \text { if } \operatorname{sh} Q=\operatorname{sh} Q^{\prime} \\ 0 & \text { otherwise. }\end{cases}
$$

Remark. $\pi_{\bar{n}}$ is the usual Robinson-Schensted correspondence. For simplification, we denote by $Z_{Q}=Z_{Q}^{(n)}$ if $Q \in \mathcal{S B T}(n)$.

In [5. Section 3], the authors give an another way to define the equivalence relation associated to this partition which looks like coplactic equivalence or dualKnuth equivalence. If $w, w^{\prime} \in W_{D}$, we write $w \smile_{D} w^{\prime}$ if $w^{\prime} w^{-1} \in S_{D^{-}} \subset S_{\bar{n}}=$ $\left\{s_{1}, \ldots, s_{n-1}\right\}$ and $\mathcal{D}_{D}^{\prime}\left(w^{-1}\right) \not \subset \mathcal{D}_{D}^{\prime}\left(w^{\prime-1}\right)$ and $\mathcal{D}_{D}^{\prime}\left(w^{\prime-1}\right) \not \subset \mathcal{D}_{D}^{\prime}\left(w^{-1}\right)$. Note that the relation $\smile_{D}$ is symmetric. We denote by $\sim_{D}$ the reflexive and transitive closure of $\smile_{D}$. It is an equivalence relation, called the coplactic equivalence relation. The equivalence classes for this relation are called the coplactic classes of $W_{D}$. We denote by $\operatorname{Cop}\left(W_{D}\right)$ the set of coplactic classes for the relation $\sim_{D}$.

By [5. Proposition 3.8], we have, for every $w, w^{\prime} \in W_{D}$,

$$
w \sim_{D} w^{\prime} \Longleftrightarrow \boldsymbol{Q}_{D}(w)=\boldsymbol{Q}_{D}\left(w^{\prime}\right) .
$$

So $\operatorname{Cop}\left(W_{D}\right)=\left\{Z_{Q}^{D} \mid Q \in \mathcal{S B T}(D)\right\}$.

Remark 4.4 - The relation $\smile_{D}$ has a useful combinatorial interpretation (see 5 , Proof of Proposition 3.8]): $w \smile_{D} s_{i} w\left(s_{i} \in S_{D^{-}}\right)$if and only if:

- either $\operatorname{sign}(w(i)) \neq \operatorname{sign}(w(i+1))$;

- or $\operatorname{sign}(w(i))=\operatorname{sign}(w(i+1))$, then $s_{i} w$ is obtained from $w$ by a classical dual-Knuth transformation; that is, $w^{-1}(i-1)$ or $w^{-1}(i+2)$ lie between $w^{-1}(i)$ and $w^{-1}(i+1)$.

Proposition 4.5. Let $w, w^{\prime} \in W_{D}$, then $w \sim_{D} w^{\prime} \Rightarrow \mathcal{D}_{D}^{\prime}(w)=\mathcal{D}_{D}^{\prime}\left(w^{\prime}\right)$. 
Proof. We may, and we will, assume that $D=(n)$. If $T_{0}$ is a Young tableau, let $\mathcal{D}\left(T_{0}\right)=\left\{s_{p} \in S_{\bar{n}} \mid p+1\right.$ lies in a row above the row containing $\left.p\right\}$. Let ${ }^{t} T_{0}$ denote the transposed tableau of $T$. If $T=\left(T^{+}, T^{-}\right)$is a standard bitableau, we set

$$
\mathcal{D}^{\prime}(T)=\left\{t_{p} \mid p \in T^{-}\right\} \uplus\left\{s_{p} \mid p \in T^{+} \text {and } p+1 \in T^{-}\right\} \uplus \mathcal{D}\left(T^{+}\right) \uplus \mathcal{D}\left({ }^{t} T^{-}\right) .
$$

Then it is easy to check that $\mathcal{D}_{n}^{\prime}(w)=\mathcal{D}^{\prime}(\boldsymbol{Q}(w))$. This completes the proof of the proposition.

Example 4.6 - Let

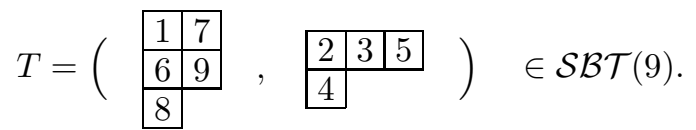

Then

$$
\mathcal{D}^{\prime}(T)=\left\{s_{1}, s_{3}, s_{6}, s_{8}, t_{2}, t_{3}, t_{4}, t_{5}\right\}
$$

Remark 4.7 - Using Proposition 4.5 we may assign a signed composition $C(Q) \|=n$ to any standard bitableau $Q \in \mathcal{S B T}(n)$ by setting $\boldsymbol{C}(Q)=\boldsymbol{C}(w)$ for any $w \in W_{n}$ such that $\boldsymbol{Q}(w)=Q$. One can determine $\boldsymbol{C}(Q)$ directly from $Q$ thanks to the following procedure, which is a combinatorial translation of the proof of Proposition 4.5 First one looks for maximal subwords $j j+1 j+2 \ldots k$ of $123 \ldots n$ such that

- either the numbers $j, j+1, j+2, \ldots, k$ can be found in this order in $Q^{+}$ when one goes from left to right (changes of rows are allowed)

- or they can be found in this order in $Q^{-}$when one goes from top to bottom (changes of column are allowed).

The word $123 \ldots n$ is then the concatenation of these maximal subwords, and the signed composition $\boldsymbol{C}(Q)$ is the sequence of the lengths of these subwords, adorned with a minus sign if the letters of the subword can be found in $Q^{-}$. As an example, consider $Q=\left(Q^{+}, Q^{-}\right)$with

$$
Q^{+}=\begin{array}{|l|l|l|l|l|l|}
\hline 1 & 2 & 6 & 7 & 8 & 13 \\
\hline 9 & 11 & 12 & &
\end{array} \quad \text { and } \quad Q^{-}=\begin{array}{|l|l|l|}
\hline 3 & 14 \\
\hline 10 & &
\end{array}
$$

The partition of $12 \ldots 1415$ in maximal subwords is $12|345| 678|9|$ $10111213 \mid 1415$, from what we can deduce that $\boldsymbol{C}(Q)=(2, \overline{3}, 3,1,4, \overline{2})$.

Therefore, we have by definitions

$$
X_{C}=\coprod_{C \leftarrow C(Q)} Z_{Q}
$$

Proposition 4.8. Let $C, D \|=n$ such that $C \subset D$. Let $w, w^{\prime} \in W_{C}$ and $x, x^{\prime} \in X_{C}^{D}$, then:

(a) If $w \sim_{C} w^{\prime}$, then $w x^{-1} \sim_{D} w^{\prime} x^{-1}$.

(b) If $x w \sim_{D} x^{\prime} w^{\prime}$, then $w \sim_{C} w^{\prime}$.

(c) If $w \sim_{C} w^{\prime}$, then $w_{C} w \sim_{C} w_{C} w^{\prime}$ and $w w_{C} \sim_{C} w^{\prime} w_{C}$. 
Proof. (c) is clear. Let us now prove (a). We may assume that $w \smile_{C} w^{\prime}$. But $\mathcal{D}_{C}\left(w^{-1}\right) \subset \mathcal{D}_{D}\left(x w^{-1}\right)$ and $\mathcal{D}_{C}\left(w^{\prime-1}\right) \subset \mathcal{D}_{D}\left(x w^{\prime-1}\right)$. So $w x^{-1} \smile_{D} w^{\prime} x^{-1}$.

We now prove (b). If $W_{C}$ is a standard parabolic subgroup of $W_{D}$, and using the fact that coplactic classes are left cells for a particular choice of parameters [5, Theorem 7.7], then (b) follows from [8]. Therefore, by taking direct products and by arguing by induction on $\left|X_{C}^{D}\right|$, we may now assume that $D=(n)$ and $C=(k, l)$ with $k, l \geq 1$ and $k+l=n$.

Let us start by proving (a). We may assume that $w \smile_{C} w^{\prime}$. But $\mathcal{D}_{C}\left(w^{-1}\right) \subset$ $\mathcal{D}_{D}\left(x w^{-1}\right)$ and $\mathcal{D}_{C}\left(w^{\prime-1}\right) \subset \mathcal{D}_{D}\left(x w^{-1}\right)$. So $w x^{-1} \smile_{D} w^{\prime} x^{-1}$.

Let us now prove (b). We may assume that $x w \smile_{D} x^{\prime} w^{\prime}$. Let $Q=\boldsymbol{Q}_{C}(w)=$ $\boldsymbol{Q}_{C}\left(w^{\prime}\right)$. From Remark 4.4 we have two cases: either $x^{\prime} w^{\prime}$ is obtained from $x w$ by a dual-Knuth relation, or $x^{\prime} w^{\prime}=s_{i} x w$ and $\operatorname{sign}(x w(i)) \neq \operatorname{sign}(x w(i+1))$. In the first case, observe that, for any $i \in[1, k-1]$ and $i \in[k+1, k+l-1], x w(k)<x w(k+1)$ if and only if $w(k)<w(k+1)$, since $x \in X_{(k, l)}=X_{(\bar{k}, \bar{l})}^{(\bar{n})} \subset W_{\bar{n}}$. Then we conclude by Remark 4.4 (which is exactly the result of Lascoux and Schützenberger on the shuffle of plactic classes [13]).

In the second case, observe that, for any $k \in[1, n]$,

$$
(\star) \quad \operatorname{sign}(w(k))=\operatorname{sign}(x w(k))=\operatorname{sign}\left(s_{i} x w(k)\right)
$$

since $X_{(k, l)}=X_{(\bar{k}, \bar{l})}^{(\bar{n})} \subset W_{\bar{n}}$ and $s_{i} \in S_{\bar{n}}$. If $s_{i} x=x^{\prime}$, then $w=w^{\prime}$ and the result follows. If $s_{i} x=x s_{j}$, with $s_{j} \in S_{(\bar{k}, \bar{l})}$ (by Deodhar's Lemma), then $x^{\prime}=x$. Therefore $w^{\prime}=s_{j} w$, by $(\star)$ and Remark 4.4 So $w \smile_{C} w^{\prime}$.

4.3. Coplactic space. Let $D \Vdash n$. If $Q \in \mathcal{S B T}(D)$, we set

$$
z_{Q}^{D}=\sum_{w \in Z_{Q}^{D}} w \quad \in \mathbb{Z} W_{D}
$$

Now, let

$$
\mathcal{Q}_{D}=\underset{Q \in \mathcal{S B T}(D)}{\oplus} \mathbb{Z} z_{Q}^{D} \quad \subset \mathbb{Z} W_{D}
$$

and

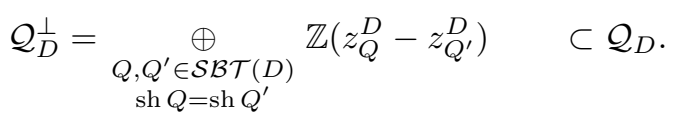

Then, by Proposition 4.5, we have

$$
\Sigma^{\prime}\left(W_{D}\right) \subset \mathcal{Q}_{D}
$$

The next proposition justifies the notation $\mathcal{Q}_{D}^{\perp}$ :

Proposition 4.10. $\mathcal{Q}_{D}^{\perp}=\left\{x \in \mathcal{Q}_{D} \mid \forall y \in \mathcal{Q}_{D}, \tau_{D}(x y)=0\right\}$.

Proof. Let $\mathcal{Q}_{D}^{\prime}=\left\{x \in \mathcal{Q}_{D} \mid \forall y \in \mathcal{Q}_{D}, \tau_{D}(x y)=0\right\}$. Let $Q$ and $Q^{\prime}$ be elements of $\mathcal{S B T}(D)$. Then, by 4.2 we have

$$
\tau_{D}\left(z_{Q}^{D} z_{Q^{\prime}}^{D}\right)= \begin{cases}1 & \text { if } \operatorname{sh} Q=\operatorname{sh} Q^{\prime} \\ 0 & \text { otherwise. }\end{cases}
$$

This shows in particular that $\mathcal{Q}_{D}^{\perp} \subset \mathcal{Q}_{D}^{\prime}$.

Let us now prove that $\mathcal{Q}_{D}^{\prime} \subset \mathcal{Q}_{D}^{\perp}$. Now, since $\mathcal{Q}_{D} / \mathcal{Q}_{D}^{\perp}$ is torsion free, it is sufficient to prove that $\operatorname{dim}_{\mathbb{Q}} \mathbb{Q} \mathcal{Q}_{D}^{\prime} \leq \operatorname{dim}_{\mathbb{Q}} \mathbb{Q} \mathcal{Q}_{D}^{\perp}$. But, by construction, we have 
$\operatorname{dim}_{\mathbb{Q}} \mathbb{Q} \mathcal{Q}_{D}-\operatorname{dim}_{\mathbb{Q}} \mathbb{Q} \mathcal{Q}_{D}^{\perp}=|\operatorname{Bip}(D)|$. Moreover, by Proposition 3.8 we have $\operatorname{dim}_{\mathbb{Q}} \mathbb{Q} \mathcal{Q}_{D}-\operatorname{dim}_{\mathbb{Q}} \mathbb{Q} \mathcal{Q}_{D}^{\prime} \geq\left|\operatorname{Irr} W_{D}\right|=|\operatorname{Bip}(D)|$.

The next lemma is a generalization to our case of a result of Blessenohl and Schocker concerning the symmetric group 3 .

Proposition 4.12. We have $\mathcal{Q}_{D}=\Sigma^{\prime}\left(W_{D}\right)+\mathcal{Q}_{D}^{\perp}$ and $\Sigma^{\prime}\left(W_{D}\right) \cap \mathcal{Q}_{D}^{\perp}=\operatorname{Ker} \theta_{D}$.

Proof. Let us first prove that $\mathcal{Q}_{D}=\Sigma^{\prime}\left(W_{D}\right)+\mathcal{Q}_{D}^{\perp}$. For this, we may, and we will, assume that $D=(n)$. We first need to introduce an order on bipartitions of $n$. We denote by $\leq_{\mathrm{s} 1}$ the lexicographic order on $\operatorname{Bip}(n)$ induced by the following order on $I_{n}$ :

$$
\overline{1}<_{\mathrm{sl}} \overline{2}<_{\mathrm{sl}} \cdots<_{\mathrm{sl}} \bar{n}<_{\mathrm{sl}} 1<_{\mathrm{sl}} 2<_{\mathrm{sl}} \cdots<_{\mathrm{sl}} n .
$$

If $\lambda$ is a bipartition of $n$, we denote by $Q_{\lambda}=Q\left(\eta_{\hat{\lambda}}\right)$. If $\lambda=\left(\lambda^{+}, \lambda^{-}\right)$, then it is easy to check that $\operatorname{sh} Q_{\lambda}=\left(\lambda^{+},{ }^{t} \lambda^{-}\right)=\lambda^{*}$, where ${ }^{t} \lambda^{-}$is the transpose of the partition $\lambda$, and, using Remarque 4.7 that $Q_{\lambda}$ is obtained by numbered $Q_{\lambda}^{+}$(resp. ${ }^{t} Q_{\lambda}^{-}$) the first column first, then the second one and so on. Now, let $Q \in \mathcal{S} \mathcal{B} \mathcal{T}(n)$. Then:

Lemma 4.13. Assume that $Z_{Q} \subset X_{\hat{\lambda}}$, then $\lambda \leq_{\mathrm{sl}}(\operatorname{sh} Q)^{*}$. Moreover, if $\operatorname{sh} Q=\lambda^{*}$, then $Q=Q_{\lambda}$.

Proof. First, we easily check (using Remark 4.7), that $\boldsymbol{\lambda}(\boldsymbol{C}(Q)) \leq_{\mathrm{sl}}$ $(\operatorname{sh} Q)^{*}$ with equality if and only if $Q=Q_{\lambda}$.

Then, observe (using Theorem 3.15), that $\lambda \leq_{\text {sl }} \boldsymbol{\lambda}(\boldsymbol{C}(Q))$ with equality if and only if $\boldsymbol{C}(Q)=\hat{\lambda}$. This conclude the proof.

We are now ready to prove by descending induction on $(\operatorname{sh} Q)^{*}$ that $z_{Q} \in$ $\Sigma^{\prime}\left(W_{n}\right)+\mathcal{Q}_{n}^{\perp}$. If $(\operatorname{sh} Q)^{*}=(n, \emptyset)$, then $Z_{Q}=\{1\}=Y_{n}$. So $z_{Q}=y_{n} \in \Sigma^{\prime}\left(W_{n}\right)$.

Now, assume that $(\operatorname{sh} Q)^{*}<_{\mathrm{sl}}(n, \emptyset)$ and that $z_{Q^{\prime}} \in \Sigma^{\prime}\left(W_{n}\right)+\mathcal{Q}_{n}^{\perp}$ for every $Q^{\prime} \in \mathcal{S B T}(n)$ such that $(\operatorname{sh} Q)^{*}<_{\mathrm{sl}}\left(\operatorname{sh} Q^{\prime}\right)^{*}$. Let $\lambda=(\operatorname{sh} Q)^{*}$. Then $z_{Q}=z_{Q_{\lambda}}+$ $\left(z_{Q}-z_{Q_{\lambda}}\right) \in z_{Q_{\lambda}}+\mathcal{Q}_{n}^{\perp}$. So it is sufficient to prove that $z_{Q_{\lambda}} \in \Sigma^{\prime}\left(W_{n}\right)+\mathcal{Q}_{n}^{\perp}$. But, by Lemma $4.13 x_{\hat{\lambda}}-z_{Q_{\lambda}}$ is a sum of $z_{Q^{\prime}}$ with $\lambda<_{\operatorname{lex}}(\operatorname{sh} Q)^{*}$. Hence, by the induction hypothesis, we have $x_{\lambda}-z_{Q_{\hat{\lambda}}} \in \Sigma^{\prime}\left(W_{n}\right)+\mathcal{Q}_{n}^{\perp}$, as desired.

Now, let us prove that $\Sigma^{\prime}\left(W_{D}\right) \cap \mathcal{Q}_{D}^{\perp}=\operatorname{Ker} \theta_{D}$. The natural map $\Sigma^{\prime}\left(W_{D}\right) \rightarrow$ $\mathcal{Q}_{D} / \mathcal{Q}_{D}^{\perp}$ is surjective, so $\operatorname{rank}_{\mathbb{Z}} \Sigma^{\prime}\left(W_{D}\right) \cap \mathcal{Q}_{D}^{\perp}=\operatorname{rank}_{\mathbb{Z}} \operatorname{Ker} \theta_{D}$. Since the $\mathbb{Z}$-modules $\Sigma^{\prime}\left(W_{D}\right) /\left(\Sigma^{\prime}\left(W_{D}\right) \cap \mathcal{Q}_{D}^{\perp}\right)$ and $\Sigma^{\prime}\left(W_{D}\right) / \operatorname{Ker} \theta_{D}$ are torsion free, it is sufficient to prove that $\Sigma^{\prime}\left(W_{D}\right) \cap \mathcal{Q}_{D}^{\perp}$ is contained in $\operatorname{Ker} \theta_{D}$. But this follows from Proposition 4.10 and Corollary 3.9

Using Proposition 4.12 we can easily extend the linear map $\theta_{D}$ to a linear map $\tilde{\theta}_{D}: \mathcal{Q}_{D} \rightarrow \mathbb{Z} \operatorname{Irr} W_{D}$. If $x \in \mathcal{Q}_{D}$, write $x=a+b$ with $a \in \Sigma^{\prime}\left(W_{D}\right)$ and $b \in \mathcal{Q}_{D}^{\perp}$ and set

$$
\tilde{\theta}_{D}(x)=\theta_{D}(a) .
$$

Then Proposition 4.12 shows that $\tilde{\theta}_{D}$ is well-defined (that is, $\theta_{D}(a)$ does not depend on the choice of $a$ and $b$ ).

Theorem 4.14. Let $D \| n$. Then: 
(a) $\tilde{\theta}_{D}$ is an extension of $\theta_{D}$ to $\mathcal{Q}_{D}$;

(b) $\operatorname{Ker} \tilde{\theta}_{D}=\mathcal{Q}_{D}^{\perp}$;

(c) if $x$ and $y$ are two elements of $\mathcal{Q}_{D}$, then $\tau_{D}(x y)=\left\langle\tilde{\theta}_{D}(x), \tilde{\theta}_{D}(y)\right\rangle_{D}$;

(d) the diagram

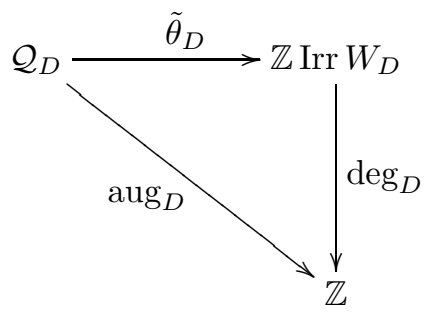

is commutative;

(e) if $x \in \mathcal{Q}_{D}$, then

$$
\tilde{\theta}_{D}(x)=\left|W_{D}\right| \sum_{\lambda \in \operatorname{Bip}(D)} \frac{\tau_{D}\left(x E_{\lambda}^{D}\right)}{\left|\mathcal{C}_{\lambda}^{D}\right|} f_{\lambda}^{D} .
$$

Proof. (a) and (b) are easy. (c) follows from Proposition 3.8 and Proposition 4.10 (d) follows from the commutativity of the diagram [3.6 and from the fact that $\operatorname{aug}_{D}\left(\mathcal{Q}_{D}^{\perp}\right)=0$ (indeed, if $Q$ and $Q^{\prime}$ are two elements of $\mathcal{S B T}(D)$ of the same shape, then $\left.\left|Z_{Q}^{D}\right|=\left|Z_{Q^{\prime}}^{D}\right|\right)$. Using Proposition 4.12 it is sufficient to prove (e) for $x \in \Sigma^{\prime}\left(W_{D}\right)$ or $x \in \mathcal{Q}_{D}^{\perp}$. If $x \in \Sigma^{\prime}\left(W_{D}\right)$, this follows from Proposition [3.10] If $x \in \mathcal{Q}_{D}^{\perp}$, this follows from Proposition 4.10 .

Remark. In the theorem, the case $D=(\bar{n})$ is precisely the symmetric group case.

Corollary 4.15. If $\tilde{\theta}$ is an extension of $\theta_{D}$ to the $\mathcal{Q}_{D}$ such that, for all $x$ and $y$ in $\mathcal{Q}_{D}, \tau_{D}(x y)=\langle\tilde{\theta}(x), \tilde{\theta}(y)\rangle_{D}$, then $\tilde{\theta}=\tilde{\theta}_{D}$.

Proof. Assume that $\tilde{\theta}$ is an extension of $\theta_{D}$ to $\mathcal{Q}_{D}$ such that $\tau_{D}(x y)=\langle\tilde{\theta}(x), \tilde{\theta}(y)\rangle_{D}$ for all $x$ and $y$ in $\mathcal{Q}_{D}$. Then, if $x \in \mathcal{Q}_{D}^{\perp}$ and $\chi \in \mathbb{Z} \operatorname{Irr} W_{D}$, then there exists $y \in \mathcal{Q}_{D}$ such that $\tilde{\theta}_{D}(y)=\chi$. So

$$
\langle\chi, \tilde{\theta}(x)\rangle_{D}=\langle\tilde{\theta}(y), \tilde{\theta}(x)\rangle_{D}=\tau_{D}(x y)=0
$$

by hypothesis and by Proposition 4.10 Since $\langle., .\rangle_{D}$ is a perfect pairing on $\mathbb{Z} \operatorname{Irr} W_{D}$, we get that $\tilde{\theta}(x)=0$. So $\tilde{\theta}$ coincides with $\tilde{\theta}_{D}$ on $\Sigma^{\prime}\left(W_{D}\right)$ and on $\mathcal{Q}_{D}^{\perp}$, so $\tilde{\theta}=\tilde{\theta}_{D}$ by Proposition 4.12

Let $\lambda \in \operatorname{Bip}(D)$. Let $Q \in \mathcal{S B T}(D)$ be of shape $\lambda$. Now, let

$$
\xi_{\lambda}=\tilde{\theta}_{D}\left(z_{Q}\right) .
$$

Then $\xi_{\lambda}$ depends only on $\lambda$ and not on the choice of $Q$. Moreover, $\xi_{\lambda} \in \mathbb{Z} \operatorname{Irr} W_{D}$, $\operatorname{deg}_{D} \xi_{\lambda}=\left|Z_{Q}\right|>0$ (see Theorem 4.14(d)) and, by Theorem 4.14 (c) and 4.11] we have $\left\langle\xi_{\lambda}, \xi_{\lambda}\right\rangle_{D}=1$. This shows that $\xi_{\lambda} \in \operatorname{Irr} W_{D}$. So we have proved the following proposition:

Proposition 4.16. The map $\operatorname{Bip}(D) \rightarrow \operatorname{Irr} W_{D}, \lambda \mapsto \xi_{\lambda}$ is well-defined and bijective. 
Remark 4.17 - If $T=\left(T^{+}, T^{-}\right) \in \mathcal{S B T}(n)$, we denote by $T^{\vee}$ the standard bitableau $\left(T^{-}, T^{+}\right)$. If $\lambda=\left(\lambda^{+}, \lambda^{-}\right) \in \operatorname{Bip}(n)$, we set $\lambda^{\vee}=\left(\lambda^{-}, \lambda^{+}\right) \in \operatorname{Bip}(n)$. In particular, $\operatorname{sh} T^{\vee}=(\operatorname{sh} T)^{\vee}$.

Now, let $w \in W_{n}$. Then $\pi_{n}\left(w_{n} w\right)=\left(\boldsymbol{P}(w)^{\vee}, \boldsymbol{Q}(w)^{\vee}\right)$. Therefore, if $Q \in \mathcal{S B T}(n)$ then $w_{n} Z_{Q}=Z_{Q^{\vee}}$. This shows in particular that $w_{n} \mathcal{Q}_{n}=\mathcal{Q}_{n}$ and that $w_{n} \mathcal{Q}_{n}^{\perp}=$ $\mathcal{Q}_{n}^{\perp}$. Moreover,

$$
\tilde{\theta}_{n}\left(w_{n} z\right)=\varepsilon_{n} \tilde{\theta}_{n}(z)
$$

for all $z \in \mathcal{Q}_{n}$. Indeed, this equality is true if $z \in \Sigma^{\prime}\left(W_{n}\right)$ by Theorem 3.7 and it is obviously true if $z \in \mathcal{Q}_{n}^{\perp}$. So we can conclude using Proposition 4.12 In particular, if $\lambda \in \operatorname{Bip}(n)$, then

$$
\xi_{\lambda \vee}=\varepsilon_{n} \xi_{\lambda} .
$$

Remark 4.20 - Let $Q \in \mathcal{S B T}(n)$ be such that $Q^{-}=\emptyset$. Then $z_{Q} \in \mathcal{Q}_{\bar{n}}$. Therefore, $\mathcal{Q}_{\bar{n}} \subset \mathcal{Q}_{n}$. Moreover, $\mathcal{Q}_{\bar{n}}^{\perp}=\mathcal{Q}_{n}^{\perp} \cap \mathcal{Q}_{\bar{n}}$. Therefore, it follows from the commutativity of Diagram 3.4 that the diagram

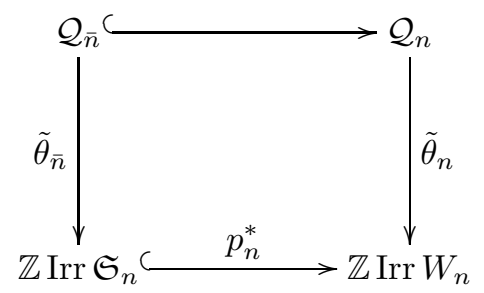

is commutative. In particular, if $\lambda=\left(\lambda^{+}, \emptyset\right)$ is the shape of $Q$, and if we denote by $\chi_{\lambda^{+}}^{\bar{n}}$ the irreducible character of $\mathfrak{S}_{n}$ associated to $\lambda^{+}$(apply Proposition 4.16 with $D=(\bar{n}))$, we have

$$
\xi_{\lambda}=p_{n}^{*} \chi_{\lambda^{+}}^{\bar{n}}
$$

4.4. Induction. We first start by an easy consequence of Proposition 4.8

Lemma 4.23. Let $C, D \| n$ be such that $C \subset D$. Let $x \in \mathcal{Q}_{C}$. Then

(a) $x_{C}^{D} x \in \mathcal{Q}_{D}$.

(b) If $x \in \mathcal{Q}_{C}^{\perp}$, then $x_{C}^{D} x \in \mathcal{Q}_{D}^{\perp}$.

Proof. (a) By linearity, we may assume that $x=z_{Q}^{C}$ with $Q \in \mathcal{S B T}(C)$. Then, by Proposition 4.8 (a), we have that $X_{C}^{D} \cdot Z_{Q}^{C}$ is a union of coplactic classes. So $x_{C}^{D} x \in \mathcal{Q}_{D}$.

(b) By linearity, we may assume that $x=z_{Q}^{C}-z_{Q^{\prime}}^{C}$ where $Q, Q^{\prime} \in \mathcal{S B T}(C)$ and $\operatorname{sh}(Q)=\operatorname{sh}\left(Q^{\prime}\right)$. We denote by $\psi: Z_{Q}^{C} \rightarrow Z_{Q^{\prime}}^{C}$ the unique bijection such that $\boldsymbol{P}_{C}(\psi(w))=\boldsymbol{P}_{C}(w)$ for every $w \in Z_{Q}^{C}$.

Then $x_{C}^{D} x=x_{C}^{D} \cdot \sum_{w \in Z_{Q}^{C}}(w-\psi(w))$. But, if $a \in X_{C}^{D}$ and $w \in Z_{Q}^{C}$, then $\boldsymbol{P}_{D}(a w)=\boldsymbol{P}_{D}(a \psi(w))$ by Proposition $4.8(\mathrm{~b})$ and 4.1 We set $\psi^{\prime}(a w)=a \psi(w)$, then the map $\psi^{\prime}: X_{C}^{D} \cdot Z_{Q}^{C} \rightarrow X_{C}^{D} \cdot Z_{Q^{\prime}}^{C}$ is bijective and satisfies $\operatorname{sh} \boldsymbol{Q}_{D}\left(\psi^{\prime}(w)\right)=$ $\operatorname{sh} \boldsymbol{Q}_{D}(w)$ for every $w \in X_{C}^{D} \cdot Z_{Q}^{C}$.

Now, let $\lambda \in \operatorname{Bip}(D)$ and let $\mathcal{E}_{\lambda}$ (resp. $\mathcal{E}_{\lambda}^{\prime}$ ) be the set of $w \in X_{C}^{D} . Z_{Q}^{C}$ (resp. $\left.w \in X_{C}^{D} \cdot Z_{Q^{\prime}}^{C}\right)$ such that $\operatorname{sh} \boldsymbol{Q}_{D}(w)=\lambda$. Then $\psi^{\prime}$ induces a bijection between 
$\mathcal{E}_{\lambda}$ and $\mathcal{E}_{\lambda}^{\prime}$. Write $\mathcal{E}_{\lambda}=\coprod_{i=1}^{r} Z_{Q_{i}}^{D}$ and $\mathcal{E}_{\lambda}^{\prime}=\coprod_{i=1}^{r^{\prime}} Z_{Q_{i}^{\prime}}^{D}$, using (a). Then, since $\left|Z_{Q_{1}}^{D}\right|=\cdots=\left|Z_{Q_{r}}^{D}\right|=\left|Z_{Q_{1}^{\prime}}^{D}\right|=\cdots=\left|Z_{Q_{r^{\prime}}}^{D}\right|$ and $\left|\mathcal{E}_{\lambda}\right|=\left|\mathcal{E}_{\lambda}^{\prime}\right|$, we have $r=r^{\prime}$. This shows that $x_{C}^{D} x \in \mathcal{Q}_{D}^{\perp}$.

Corollary 4.24. Let $C, D \| n$ be such that $C \subset D$. Then the diagram

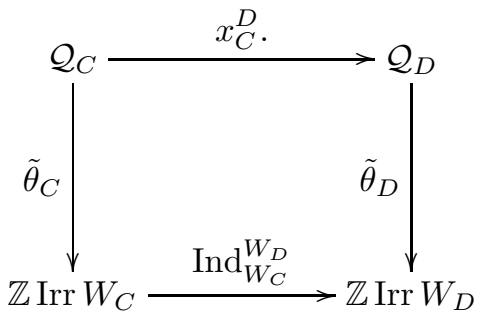

is commutative.

Proof. This follows immediately from Proposition 4.23 and from the commutativity of the diagram 3.3

Now, if $\lambda \in \operatorname{Bip}(n)$, then we denote by $\chi_{\lambda}$ the irreducible character of $W_{n}$ associated to $\lambda$ via Clifford theory (see 9 ). The link between the two parametrizations (the $\xi$ 's and the $\chi$ 's) is given by the following result:

Corollary 4.25. If $\lambda$ is a bipartition of $n$, then $\xi_{\lambda}=\chi_{\lambda^{*}}$.

Proof. Write $\lambda=\left(\lambda^{+}, \lambda^{-}\right), k=\left|\lambda^{+}\right|$and $l=\left|\lambda^{-}\right|$. Let $Q^{+}$be a standard tableau of shape $\lambda^{+}$filled with $\{l+1, l+2, \ldots, n\}$ and let $Q^{-}$be a standard tableau of shape $\lambda^{-}$filled with $\{1,2, \ldots, l\}$. Then, by [5, Proposition 4.8],

$$
Z_{Q}=X_{l, k}\left(w_{l} Z_{Q^{-}} \times Z_{Q^{+}}\right) .
$$

Therefore, by Corollary 4.24 we have

$$
\xi_{\lambda}=\operatorname{Ind}_{W_{l, k}}^{W_{n}}\left(\tilde{\theta}_{l}\left(w_{l} Z_{Q^{-}}^{l}\right) \otimes \tilde{\theta_{k}}\left(Z_{Q^{+}}^{k}\right)\right) .
$$

So, by 4.22 and by Remark 4.17 we have

$$
\xi_{\lambda}=\operatorname{Ind}_{W_{k, l}}^{W_{n}}\left(p_{k}^{*} \chi_{\lambda^{+}}^{\bar{k}} \otimes \varepsilon_{l}\left(p_{l}^{*} \chi_{\lambda^{-}}^{\bar{l}}\right)\right) .
$$

The result now follows from 9 .

\section{Related Hopf algebras}

5.1. Hopf algebra of signed permutations. Consider the graded $\mathbb{Z}$-module

$$
\mathcal{S P}=\underset{n \geq 0}{\oplus} \mathbb{Z} W_{n}
$$

where $W_{0}=1$. In [1], Aguiar and Mahajan have shown that $\mathcal{S P}$ has a structure of Hopf algebra which is similar to the structure of the Malvenuto-Reutenauer Hopf algebra on permutations [15. Moreover, they have shown that

$$
\Sigma^{\prime}=\underset{n \geq 0}{\oplus} \Sigma^{\prime}\left(W_{n}\right)
$$

is a Hopf subalgebra of $\mathcal{S P}$. We revise here the definition of the product and the coproduct on $\mathcal{S P}$ with our point of view. 
Notation - If $C$ is a signed composition, then we denote by $x_{C}$ the element of $\mathcal{S P}$ lying in $\mathbb{Z} W_{|C|}$ corresponding to the $x_{C}$ defined in $\$ 3$ Similarly, if $Q$ is a standard bitableau, then $z_{Q} \in \mathbb{Z} W_{|\operatorname{sh} Q|}$ is viewed as an element of $\mathcal{S P}$.

Let $(u, v) \in W_{n} \times W_{m}$, we denote $u \times v$ the corresponding element of $W_{n, m} \simeq$ $W_{n} \times W_{m}$. If $w \in W n, m$, we denote by $\left(w_{(n)}^{\prime}, w_{(m)}^{\prime \prime}\right)$ the corresponding element of $W_{n} \times W_{m}$. We now define

$$
u * v=x_{n, m}(u \times v) \in \mathbb{Z} W_{n+m} .
$$

We extend $*$ by linearity to a bilinear map $\mathcal{S P} \times \mathcal{S P} \rightarrow \mathcal{S P}$.

Now, let $w \in W_{n}$. Then, for each $i \in[0, n]$, we denote by $\pi_{i}(w)$ the unique element of $W_{i, n-i}$ such that $w \in \pi_{i}(w) X_{i, n-i}^{-1}$. We set

$$
\Delta(w)=\sum_{i=0}^{n} \pi_{i}(w)_{(i)}^{\prime} \otimes_{\mathbb{Z}} \pi_{i}(w)_{(n-i)}^{\prime \prime} \in \mathcal{S P} \otimes \mathcal{S P}
$$

We extend $\Delta$ by linearity to a map $\Delta: \mathcal{S P} \rightarrow \mathcal{S P} \otimes_{\mathbb{Z}} \mathcal{S P}$.

Remark 5.1 - Combinatorially, we see this product as follows: let $w=w_{1} \ldots w_{n}$ be a word of length $n$ in the alphabet $I_{n}$, the standardsigned permutation is the unique element $\operatorname{sts}(w) \in W_{n}$ such that

$$
\left\{\begin{array}{l}
\operatorname{sts}(w)(i)<\operatorname{sts}(w)(j) \Leftrightarrow\left(w_{i}<w_{j}\right) \quad \text { or } \quad\left(w_{i}=w_{j} \text { and } i<j\right) \\
\text { and } \operatorname{sign}(\operatorname{sts}(w)(i))=\operatorname{sign}\left(w_{i}\right) .
\end{array}\right.
$$

Then

$$
u * v=\sum_{w, w^{\prime}} w w^{\prime}
$$

where $w w^{\prime}$ is the concatenation of $w$ and $w^{\prime}$; and the sum is taken over all words $w, w^{\prime}$ on the alphabet $I_{n}$ such that $\operatorname{sts}(w)=u, \operatorname{sts}\left(w^{\prime}\right)=v$ and alph $(u) \uplus \operatorname{alph}(v)=$ $[1, n]$ (where $\operatorname{alph}(u)=$ the set of absolute values of the letters in $u$ ). For instance, $\overline{1} 2 \times 2 \overline{1}=\overline{1} 24 \overline{3}$ and

$$
\begin{aligned}
x_{(2,2)} & =y_{(2,2)}+y_{(4)} \\
& =1234+1324+1423+2314+2413+3412 .
\end{aligned}
$$

Hence $\overline{1} 2 * 2 \overline{1}=\overline{1} 24 \overline{3}+\overline{1} 34 \overline{2}+\overline{1} 43 \overline{2}+\overline{2} 34 \overline{1}+\overline{2} 43 \overline{1}+\overline{3} 42 \overline{1}$.

Remark 5.2 - For $w \in W_{n}$ seen as a word on the alphabet $I_{n}$ and $i<j \in[1, n]$, we denote $w \mid[i, j]$ the subword obtained by taking only the digits such that their absolute values are in $[i, j]$. Then we see combinatorially the coproduct as

$$
\Delta(w)=\sum_{i=0}^{n} w \mid[1, i] \otimes \operatorname{sts}(w \mid[i+1, n]) .
$$

As example, consider $w=\overline{2} 31 \overline{4}$, then we have the following decompositions:

$$
w^{-1}=3 \overline{1} 2 \overline{4}=3124(1 \times \overline{1} 2 \overline{3})=1324(2 \overline{1} \times 1 \overline{2})=1234(3 \overline{1} 2 \times \overline{1}) .
$$

Hence

$$
w=\overline{2} 31 \overline{4}=(1 \times \overline{1} 2 \overline{3}) 2314=(\overline{2} 1 \times 1 \overline{2}) 1324=(\overline{2} 31 \times \overline{1}) 1234 .
$$

Thus

$$
\Delta(\overline{2} 31 \overline{4})=\emptyset \otimes \overline{2} 31 \overline{4}+1 \otimes \overline{1} 2 \overline{3}+\overline{2} 1 \otimes 1 \overline{2}+\overline{2} 31 \otimes \overline{1}+\overline{2} 31 \overline{4} \otimes \emptyset .
$$


Example 5.3 - Let $C$ and $D$ be two signed composition. We denote by $C \sqcup D$ the signed composition obtained by concatenation of $C$ and $D$. Then

$$
x_{C} * x_{D}=x_{C \sqcup D} .
$$

Example 5.4 - We have

$$
\begin{aligned}
& \Delta\left(x_{n}\right)=\sum_{i=0}^{n} x_{i} \otimes_{\mathbb{Z}} x_{n-i} \\
& \Delta\left(x_{\bar{n}}\right)=\sum_{i=0}^{n} x_{\bar{i}} \otimes_{\mathbb{Z}} x_{\overline{n-i}} .
\end{aligned}
$$

We state here a result of Aguiar and Mahajan 1], with our basis consisting of the $x_{C}$.

Theorem 5.5. The graded vector space $\mathcal{S P}$, with the product $*$ and the coproduct $\Delta$ is a connected graded Hopf algebra; and $\Sigma^{\prime}$ is a Hopf subalgebra of $\mathcal{S P}$ which is freely generated by elements $\left(x_{n}\right)_{n \in \mathbb{Z} \backslash\{0\}}$ as algebra.

If $x, y \in \mathcal{S P}$, we define the product $x y \in \mathcal{S P}$ as follows: if $x \in \mathbb{Z} W_{n}$ and $y \in \mathbb{Z} W_{m}$, then $x y=0$ if $m \neq n$ and $x y$ coincides with the usual product $x y$ in $\mathbb{Z} W_{n}$ if $m=n$. Let $\tau: \mathcal{S P} \rightarrow \mathbb{Z}$ be the unique linear map which coincides with $\tau_{n}$ on $\mathbb{Z} W_{n}$. The the map $\mathcal{S P} \times \mathcal{S P} \rightarrow \mathbb{Z},(x, y) \mapsto \tau(x y)$ is a scalar product on $\mathcal{S P}$. If $x, y \in \mathcal{S P}$, we set

$$
\tau_{\otimes}(x \otimes y)=\tau(x) \tau(y)
$$

The following proposition is easily checked from definitions:

Proposition 5.6. $\mathcal{S P}$ is self-dual for $\tau$, that is,

$$
\tau_{\otimes}((u \otimes v) \Delta(w))=\tau((u * v) w)
$$

for all $u, v, w \in \mathcal{S P}$.

5.2. The Hopf algebra of characters. We give here a short recall of a result of Geissinger [10]. Consider the graded $\mathbb{Z}$-module

$$
\mathcal{C H} \mathcal{A R}=\underset{n \geq 0}{\oplus} \mathbb{Z} \operatorname{Irr} W_{n} .
$$

If $k$ and $l$ are two natural numbers, we denote by $\iota_{k, l}$ the canonical isomorphism

$$
\iota_{k, l}: \mathbb{Z} \operatorname{Irr} W_{k} \otimes_{\mathbb{Z}} \mathbb{Z} \operatorname{Irr} W_{l} \stackrel{\sim}{\longrightarrow} \mathbb{Z} \operatorname{Irr} W_{k, l} .
$$

Let $(\chi, \psi) \in \mathbb{Z} \operatorname{Irr} W_{k} \times \mathbb{Z} \operatorname{Irr} W_{l}$. We define

$$
\chi \bullet \psi=\operatorname{Ind}_{W_{k, l}}^{W_{k+l}} \iota_{k, l}\left(\chi \otimes_{\mathbb{Z}} \psi\right) \quad \in \mathbb{Z} \operatorname{Irr} W_{k+l} .
$$

Now, let $\chi \in \mathcal{C} \mathcal{L}_{\mathbb{Q}} W_{n}$. We define

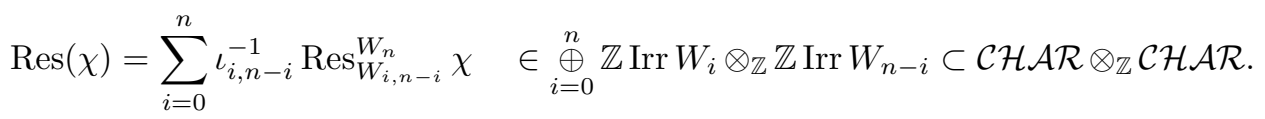

We denote $\langle\cdot, \cdot\rangle$ the unique scalar product on $\mathcal{C H} \mathcal{A R}$ which coincides with $\langle\cdot, \cdot\rangle_{n}$ on $\mathbb{Z} \operatorname{Irr} W_{n}$ and such that $\mathbb{Z} \operatorname{Irr} W_{n}$ and $\mathbb{Z} \operatorname{Irr} W_{m}$ are orthogonal if $m \neq n$. We now define $\langle\cdot, \cdot\rangle_{\otimes}$ on $\mathcal{C H} \mathcal{A R} \otimes_{\mathbb{Z}} \mathcal{C H} \mathcal{A R}$ as follows: if $\chi, \chi^{\prime}, \psi, \psi^{\prime} \in \mathcal{C H} \mathcal{A R}$, we set

$$
\left\langle\chi \otimes \psi, \chi^{\prime} \otimes \psi^{\prime}\right\rangle_{\otimes}=\left\langle\chi, \chi^{\prime}\right\rangle\left\langle\psi, \psi^{\prime}\right\rangle .
$$


Geissinger 10] has shown that $\mathcal{C H} \mathcal{A R}$ with product $\bullet$ and coproduct Res is a connected graded Hopf algebra. Moreover, for any $\chi, \psi, \zeta \in \mathcal{C H} \mathcal{A R}$, the reciprocity law of Frobenius can be viewed as

$$
\langle\chi \otimes \psi, \operatorname{Res} \zeta\rangle_{\otimes}=\langle\chi \bullet \psi, \zeta\rangle .
$$

5.3. The coplactic algebra and an Hopf epimorphism. Let us intoduce

$$
\mathcal{Q}=\underset{n \geq 0}{\oplus} \mathcal{Q}_{n}
$$

and

$$
\mathcal{C H} \mathcal{A R}=\underset{n \geq 0}{\oplus} \mathbb{Z} \operatorname{Irr} W_{n} .
$$

We define $\theta: \Sigma^{\prime} \rightarrow \mathcal{C H} \mathcal{A} \mathcal{R}$ and $\tilde{\theta}: \mathcal{Q} \rightarrow \mathcal{C H} \mathcal{A R}$ by

$$
\theta=\underset{n \geq 0}{\oplus} \theta_{n} \quad \text { and } \quad \tilde{\theta}=\underset{n \geq 0}{\oplus} \tilde{\theta}_{n} .
$$

The first part of the following theorem shows that $\mathcal{Q}$ is a generalization of the Poirier-Reutenauer Hopf algebra of tableaux [17] to our case (see also [3]), and the second part shows that Jöllenbeck's construction generalizes to our case.

Theorem 5.8. $\mathcal{Q}$ is a Hopf subalgebra of $\mathcal{S P}$ containing $\Sigma^{\prime}$. Moreover, $\theta: \Sigma^{\prime} \rightarrow$ $\mathcal{C H} \mathcal{A R}$ and $\tilde{\theta}: \mathcal{Q} \rightarrow \mathcal{C H} \mathcal{A R}$ are surjective Hopf algebra homomorphisms.

Proof. The fact that $\mathcal{Q}$ is a subalgebra of $\mathcal{S P}$ follows from Proposition 4.23. To prove that it is a subcoalgebra, we proceed as in the proof of the result of Poirier and Reutenauer [17, using Remark 4.4 let $Z$ be a coplactic class in $W_{n}, i \in[0, n]$ and $w \in Z$. Write $w=\pi_{i}(w) x$, where $x^{-1} \in X_{i, n-i}$. Let $u \in W_{i}$ such that $u \smile_{(i)}$ $\pi_{i}(w)_{(i)}^{\prime}$. As sign $\left(x^{-1} w^{-1}(k)\right)=\operatorname{sign}\left(w^{-1}(k)\right)$ and $x^{-1}(l)<x^{-1}(l+1)$, for all $l \in[1, i-1]$ and for all $l \in[i+1, n-1]$, we easily check that $\left(u \times \pi_{i}(w)_{(n-i)}^{\prime \prime}\right) x \smile_{(n)} w$, using Remark 4.4 Let $v \in W_{n-i}$ such that $v \smile_{(n-i)} \pi_{i}(w)_{(n-i)}^{\prime \prime}$, then $(u \times v) x \smile_{(n)}$ $w$ as above. Therefore

$$
\Delta\left(\sum_{w \in Z} w\right)=\sum_{i=0}^{n} \sum_{Z_{i}, Z_{n-i}}\left(\sum_{u \in Z_{i}} u\right) \otimes\left(\sum_{v \in Z_{n-i}} v\right),
$$

where $Z_{i}$ (resp. $Z_{n-i}$ ) are coplactic classes in $W_{i}$ (resp. $\left.W_{n-i}\right)$.

We now need to prove that $\tilde{\theta}$ is an homomorphism of Hopf algebras. We first need a lemma concerning the symmetric bilinear form $\beta:\left(\mathcal{Q} \otimes_{\mathbb{Z}} \mathcal{Q}\right) \times\left(\mathcal{Q} \otimes_{\mathbb{Z}} \mathcal{Q}\right) \rightarrow \mathbb{Z}$, $(x, y) \mapsto \tau_{\otimes}(x y)$. Let $\tilde{\theta}_{\otimes}=\tilde{\theta} \otimes_{\mathbb{Z}} \tilde{\theta}: \mathcal{Q} \otimes_{\mathbb{Z}} \mathcal{Q} \rightarrow \mathcal{C H} \mathcal{H} \mathcal{R} \otimes_{\mathbb{Z}} \mathcal{C H} \mathcal{A R}$. Then:

Lemma 5.9. $\operatorname{Ker} \tilde{\theta}_{\otimes}=\mathcal{Q} \otimes_{\mathbb{Z}} \operatorname{Ker} \tilde{\theta}+\operatorname{Ker} \tilde{\theta} \otimes_{\mathbb{Z}} \mathcal{Q}$ is the kernel of $\beta$.

Proof. By Theoren 4.14 (c), we have

$$
\beta(x, y)=\left\langle\tilde{\theta}_{\otimes}(x), \tilde{\theta}_{\otimes}(y)\right\rangle_{\otimes}
$$

for all $x, y \in \mathcal{Q} \otimes_{\mathbb{Z}} \mathcal{Q}$. This proves the lemma. 
Proposition [5.6 and Lemma [5.9 show that $\operatorname{Ker} \tilde{\theta}$ is an ideal and a coideal of $\mathcal{Q}$. Since $\mathcal{Q}=\Sigma^{\prime}+\operatorname{Ker} \tilde{\theta}$, it is sufficient to prove that $\theta$ is a bialgebra homomorphism. First, it is clear that $\left.\tilde{\theta}\left(x_{C} * x_{D}\right)=\tilde{(} x_{C \sqcup D}\right)$. So $\tilde{\theta}$ is an algebra homomorphism. Using this last propoerty and Theorem [5.5] it is sufficient to prove that $\tilde{\theta}_{\otimes}\left(\Delta\left(x_{n}\right)\right)=$ $\operatorname{Res}\left(\tilde{\theta}\left(x_{n}\right)\right)$ and $\tilde{\theta}_{\otimes}\left(\Delta\left(x_{\bar{n}}\right)\right)=\operatorname{Res}\left(\tilde{\theta}\left(x_{\bar{n}}\right)\right)$ But this follows easily from Example 5.4

\section{THE CASE $n=2$}

In this Section, we will give a complete description of the algebra $\Sigma^{\prime}\left(W_{2}\right)$. For simplification, we set $s=s_{1}$. Note that $t_{1}=t$ and $t_{2}=s t s$. In other words, $S_{2}^{\prime}=\{s, t, s t s\}$. Table I gives the correspondence between reduced decomposition of elements of $W_{2}$ and permutations of $I_{2}$ (if $w \in W_{2}$, we only give the couple $(w(1), w(2))$ since it determines $w$ as a permutation of $\left.I_{2}\right)$. It also gives the value of $\mathcal{U}_{2}^{\prime}(w)$ and $\boldsymbol{C}(w)$. Table II gives representatives of the conjugacy classes of $W_{2}$. Table III gives, for each signed composition $C$ of 2 , the subgroup $W_{C}$ of $W_{2}$, the set $S_{C}$, the elements $x_{C}$ and $y_{C}$ of $\mathbb{Z} W_{2}$ and also gives the value of $\mathcal{A}_{C}$. Table IV provides the decomposition of the induced characters $\operatorname{Ind}_{W_{\hat{\lambda}}}^{W_{2}} 1_{\hat{\lambda}}=\theta_{2}\left(x_{\hat{\lambda}}\right)$ as a combination of the $\xi_{\mu}$, for $\lambda \Vdash 2$. Table $\mathrm{V}$ gives the character table of $\mathbb{Q} \Sigma^{\prime}\left(W_{2}\right)$ (see Subsection 3.5). We give in Table VI a complete set of orthogonal primitive idempotents of $\mathbb{Q} \Sigma^{\prime}\left(W_{2}\right)$. Table VII gives the Cartan matrix of $\Sigma^{\prime}\left(W_{2}\right)$. As usual, the dots in the tables represent the number 0 . Note that

$$
w_{2}=s t s t=t s t s .
$$

We conclude the Section by a description of the algebra $\mathbb{Q} \Sigma^{\prime}\left(W_{2}\right)$ as a product of classical indecomposable algebras.

Convention. For avoiding the use of too many parenthesis, we have denoted by $\xi_{\hat{\lambda}}, \pi_{\hat{\lambda}}$ or $E_{\hat{\lambda}}$ the objects $\xi_{\lambda}, \pi_{\lambda}$ or $E_{\lambda}$ respectively. For instance, $\xi_{1, \overline{1}}=\xi_{((1),(1))}$ and $\pi_{2}=\pi_{((2), \emptyset)}$ and $E_{\overline{1}, \overline{1}}=E_{(\emptyset,(1,1))}$.

\begin{tabular}{|c|c|c|c|}
\hline$w$ & $(w(1), w(2))$ & $\mathcal{U}_{2}^{\prime}(w)$ & $\boldsymbol{C}(w)$ \\
\hline \hline 1 & $(1,2)$ & $\{s, t, s t s\}$ & $(2)$ \\
$s$ & $(2,1)$ & $\{t, s t s\}$ & $(1,1)$ \\
$t$ & $(\overline{1}, 2)$ & $\{s, s t s\}$ & $(\overline{1}, 1)$ \\
$s t$ & $(\overline{2}, 1)$ & $\{s, s t s\}$ & $(\overline{1}, 1)$ \\
$t s$ & $(2, \overline{1})$ & $\{t\}$ & $(1, \overline{1})$ \\
$s t s$ & $(1, \overline{2})$ & $\{t\}$ & $(1, \overline{1})$ \\
$t s t$ & $(\overline{2}, \overline{1})$ & $\{s\}$ & $(\overline{2})$ \\
$w_{2}$ & $(\overline{1}, \overline{2})$ & $\emptyset$ & $(\overline{1}, \overline{1})$ \\
\hline
\end{tabular}

\begin{tabular}{|c|c|c|}
\hline$\hat{\lambda}$ & $c_{\lambda}$ & $\left|\mathcal{C}_{\lambda}\right|$ \\
\hline$(2)$ & $s t$ & 2 \\
$(1,1)$ & $w_{2}$ & 1 \\
$(1, \overline{1})$ & $t$ & 2 \\
$(\overline{2})$ & $s$ & 2 \\
$(\overline{1}, \overline{1})$ & 1 & 1 \\
\hline
\end{tabular}

Table I. Elements

Table II. Conjugacy classes 


\begin{tabular}{|c|c|c|c|c|c|}
\hline$C$ & $W_{C}$ & $S_{C}$ & $x_{C}$ & $y_{C}$ & $\mathcal{A}_{C}$ \\
\hline \hline$(2)$ & $W_{2}$ & $\{s, t\}$ & 1 & 1 & $\{s, t, s t s\}$ \\
$(1,1)$ & $W_{1} \times W_{1}$ & $\{t, s t s\}$ & $1+s$ & $s$ & $\{t, s t s\}$ \\
$(\overline{1}, 1)$ & $\mathfrak{S}_{1} \times W_{1}$ & $\{s t s\}$ & $1+s+t+s t$ & $t+s t$ & $\{t\}$ \\
$(1, \overline{1})$ & $W_{1} \times \mathfrak{S}_{1}$ & $\{t\}$ & $1+s+t s+s t s$ & $t s+s t s$ & $\{s, s t s\}$ \\
$(\overline{2})$ & $\mathfrak{S}_{2}$ & $\{s\}$ & $1+t+s t+t s t$ & $t s t$ & $\{s, s t s\}$ \\
$(\overline{1}, \overline{1})$ & 1 & $\emptyset$ & $\sum_{w \in W_{2}} w$ & $w_{2}$ & $\emptyset$ \\
\hline
\end{tabular}

Table III. Bases of $\Sigma^{\prime}\left(W_{2}\right)$

\begin{tabular}{|c||c|c|c|c|c|}
\hline & $\xi_{2}$ & $\xi_{1,1}$ & $\xi_{1, \overline{1}}$ & $\xi_{\overline{2}}$ & $\xi_{\overline{1}, \overline{1}}$ \\
\hline \hline$\theta_{2}\left(x_{2}\right)$ & 1 & $\cdot$ & $\cdot$ & $\cdot$ & $\cdot$ \\
$\theta_{2}\left(x_{1,1}\right)$ & 1 & 1 & $\cdot$ & $\cdot$ & $\cdot$ \\
$\theta_{2}\left(x_{1, \overline{1}}\right)$ & 1 & 1 & 1 & $\cdot$ & $\cdot$ \\
$\theta_{2}\left(x_{\overline{2}}\right)$ & 1 & $\cdot$ & 1 & 1 & $\cdot$ \\
$\theta_{2}\left(x_{\overline{1}, \overline{1}}\right)$ & 1 & 1 & 2 & 1 & 1 \\
\hline
\end{tabular}

Table IV. Decomposition of induced characters

\begin{tabular}{|c||c|c|c|c|c|}
\hline & $x_{2}$ & $x_{1,1}$ & $x_{1, \overline{1}}$ & $x_{\overline{2}}$ & $x_{\overline{1}, \overline{1}}$ \\
\hline \hline$\pi_{2}$ & 1 & $\cdot$ & $\cdot$ & $\cdot$ & $\cdot$ \\
$\pi_{1,1}$ & 1 & 2 & $\cdot$ & $\cdot$ & $\cdot$ \\
$\pi_{1, \overline{1}}$ & 1 & 2 & 2 & $\cdot$ & $\cdot$ \\
$\pi_{\overline{2}}$ & 1 & $\cdot$ & $\cdot$ & 2 & $\cdot$ \\
$\pi_{\overline{1}, \overline{1}}$ & 1 & 2 & 4 & 4 & 8 \\
\hline
\end{tabular}

Table V. Character table of $\Sigma^{\prime}\left(W_{2}\right)$

Remark. Using these tables, one can check that $\theta_{2}\left(x_{\overline{2}}\right)\left(x_{1,1}\right)=6 \neq 4=\theta_{2}\left(x_{1,1}\right)\left(x_{\overline{2}}\right)$. In other words, the symmetry property (see 4]) does not hold in our case. 


$$
\begin{aligned}
E_{2} & =x_{2}-\frac{1}{2} x_{\overline{2}}-\frac{1}{4} x_{1, \overline{1}}+\frac{1}{4} x_{\overline{1}, 1}-\frac{1}{2} x_{1,1}+\frac{1}{4} x_{\overline{1}, \overline{1}} \\
E_{1,1} & =\frac{1}{2}\left(x_{1,1}-\frac{1}{2} x_{1, \overline{1}}-\frac{1}{2} x_{\overline{1}, 1}+\frac{1}{4} x_{\overline{1}, \overline{1}}\right) \\
E_{1, \overline{1}} & =\frac{1}{2}\left(x_{1, \overline{1}}-\frac{1}{2} x_{\overline{1}, \overline{1}}\right) \\
E_{\overline{2}} & =\frac{1}{2}\left(x_{\overline{2}}-\frac{1}{2} x_{\overline{1}, \overline{1}}\right) \\
E_{\overline{1}, \overline{1}} & =\frac{1}{8} x_{\overline{1}, \overline{1}}
\end{aligned}
$$

\section{Table VI. A complete set of orthogonal primitive idempotents}

We will now give the Cartan matrix of $\Sigma^{\prime}\left(W_{2}\right)$. If $\mu \in \operatorname{Bip}(2)$, we denote by $\Pi_{\mu}$ the character of the projective cover $\mathbb{Q} \Sigma^{\prime}\left(W_{2}\right) E_{\mu}$ of $\mathbb{Q}_{\mu}$. Write

$$
\Pi_{\mu}=\sum_{\mu \in \operatorname{Bip}(2)} \gamma_{\lambda \mu} \pi_{\lambda}
$$

Then $\left(\gamma_{\lambda \mu}\right)_{\lambda, \mu \in \operatorname{Bip}(2)}$ is the Cartan matrix of $\Sigma^{\prime}\left(W_{2}\right)$. It is given in the following table:

\begin{tabular}{|c|ccccc|}
\hline$\hat{\lambda} \backslash \hat{\mu}$ & $(2)$ & $(1,1)$ & $(1, \overline{1})$ & $(\overline{2})$ & $(\overline{1}, \overline{1})$ \\
\hline$(2)$ & 1 & $\cdot$ & $\cdot$ & $\cdot$ & $\cdot$ \\
$(1,1)$ & $\cdot$ & 1 & $\cdot$ & $\cdot$ & $\cdot$ \\
$(1, \overline{1})$ & $\cdot$ & $\cdot$ & 1 & 1 & $\cdot$ \\
$(\overline{2})$ & $\cdot$ & $\cdot$ & $\cdot$ & 1 & $\cdot$ \\
$(\overline{1}, \overline{1})$ & $\cdot$ & $\cdot$ & $\cdot$ & $\cdot$ & 1 \\
\hline
\end{tabular}

\section{Table VII. Cartan matrix of $\Sigma^{\prime}\left(W_{2}\right)$}

Let $E_{0}=E_{1, \overline{1}}+E_{\overline{2}}$. Then $\left(E_{2}, E_{1,1}, E_{0}, E_{\overline{2}}\right)$ is a complete set of central primitive idempotents (they are of course orthogonal). Therefore, write $A_{\omega}=\mathbb{Q} \Sigma^{\prime}\left(W_{2}\right) E_{\omega}$, for $\omega \in\{2,(1,1), 0, \overline{2}\}$. Then

$$
\mathbb{Q} \Sigma^{\prime}\left(W_{2}\right)=A_{2} \oplus A_{1,1} \oplus A_{\overline{2}} \oplus A_{0},
$$

as a sum of algebras. Morever, $A_{2} \simeq \mathbb{Q}, A_{1,1} \simeq \mathbb{Q}, A_{\overline{2}} \simeq \mathbb{Q}$. On the other hand,

$$
A_{0}=\mathbb{Q} E_{1, \overline{1}} \oplus \mathbb{Q} E_{\overline{2}} \oplus \mathbb{Q}\left(x_{1, \overline{1}}-x_{\overline{1}, 1}\right),
$$

as a vector space. Now, let $B$ be the algebra

$$
B=\left\{\left(\begin{array}{ll}
a & b \\
0 & c
\end{array}\right) \mid a, b, c \in \mathbb{Q}\right\}
$$

Then the $\mathbb{Q}$-linear map $\sigma: A_{0} \rightarrow B$ such that

$$
\sigma\left(E_{1, \overline{1}}\right)=\left(\begin{array}{ll}
1 & 0 \\
0 & 0
\end{array}\right), \quad \sigma\left(E_{\overline{2}}\right)=\left(\begin{array}{ll}
0 & 0 \\
0 & 1
\end{array}\right) \quad \text { and } \quad \sigma\left(x_{1, \overline{1}}-x_{\overline{1}, 1}\right)=\left(\begin{array}{cc}
0 & 1 \\
0 & 0
\end{array}\right)
$$


is an isomorphism of algebras. Therefore, we have an isomorphism of algebras

$$
\mathbb{Q} \Sigma^{\prime}\left(W_{2}\right) \simeq \mathbb{Q} \oplus \mathbb{Q} \oplus \mathbb{Q} \oplus B
$$

\section{REFERENCES}

[1] M. Aguiar, S. Mahajan: The Hopf algebra of signed permutations, in preparation.

[2] F. Bergeron, N. Bergeron, R. B. Howlett and D. E. Taylor: A decomposition of the descent algebra of a finite Coxeter group, J. Algebraic Combin., 1, 1992, p.23-44.

[3] D. Blessenohl, M. Schocker: Noncommutative Character Theory of Symmetric groups I, preprint, 2002.

[4] D. Blessenohl, C. Hohlweg, M. Schocker: A symmetry of the descent algebra of a finite Coxeter group, to appear in Advances in Math, 2003.

[5] C. Bonnafé, L. Iancu: Left cells in type $B_{n}$ with unequal parameters, Represent. Theory, $\mathbf{7}$, 2003, p.587-609.

[6] N. Bourbaki: Groupes et algèbres de Lie, Chap. 4-6, Hermann, 1968.

[7] P. Fleischmann: On pointwise conjugacy of distinguished coset representatives in Coxeter groups, J. Group Theory, 2002, 5, p.269 -283.

[8] M. Geck: On the induction of Kazhdan-Lusztig cells. Bull. London Math. Soc. 35, 5 (2003), 608-614.

[9] M. Geck, G. Pfeiffer: Characters of Finite Coxeter Groups and Iwahori-Hecke Algebras, London Math. Soc. Mon. New Series, 2000, 21.

[10] L. Geissinger: Hopf algebras of symmetric functions and class functions, In Comb. Represent. Groupe symétrique, Acte Table Ronde C.N.R.S. Strsbourg 1976, Lecture Notes in Math., 579, 1977, P.168-181.

[11] J.E. Humphreys: Reflection groups and Coxeter groups, 1990, Cambridge university press, 29.

[12] A. Jőllenbeck: Nichtkommutative Charaktertheorie der symmetrischen Gruppen, Bayreuth. Math. Schr., 56, 1999, p.1-41.

[13] A. Lascoux, M. P. Schützenberger: Le monö̈de plaxique., Noncommutative structures in algebra and geometric combinatorics (Naples, 1978), pp. 129-156, Quad. "Ricerca Sci.", 109, CNR, Rome, 1981.

[14] I. G. Macdonald: Symmetric functions and Hall Polynomials, Oxford Math. Mono., 1979.

[15] C. Malvenuto, C. Reutenauer: Duality between quasi-symmetric functions ans Solomon descent algebra, J. Algebra, 177, 1995, p. 967-982.

[16] R. Mantaci, C. Reutenauer: A generalization of Solomon's algebra for hyperoctahedral groups and other wreath products, Comm. in Algebra, 23(1), 1995, p.27-56.

[17] S. Poirier, C. Reutenauer: Algèbre de Hopf des tableaux, Ann. Sci. Math., Québec 19, 1996, P.79-90.

[18] L. Solomon: A Mackey formula in the group ring of a Coxeter group, J. Algebra, 41, 1976, p. $255-268$.

[19] W. Specht: Eine Verallgemeinerung der symmetrischen Gruppe, Schriften Math. Seminar Berlin, 1, 1932, p.1-32.

[20] R. P. Stanley: Some aspects of groups acting on finite posets, J. Combin. Theory Ser. A, 32, 1982, p.132-161.

[21] J. Y. Thibon: Lectures on Noncommutative Symmetric Functions, in Interaction of Combinatorics and Representation Theory, MSJ Memoirs 11, 2001, p.39-94. 


\section{Appendix: Comparison with Specht's construction}

\section{Pierre Baumann and Christophe Hohlweg}

The present text is an appendix to the article Generalized descent algebra and construction of irreducible characters of hyperoctahedral groups, by Cédric Bonnafé and the second present author. Our aim here is to relate two constructions of the irreducible characters of the hyperoctahedral groups: the one given in that article, and Specht's one [19]. Meant as a sequel to Bonnafé and Hohlweg's article, this text uses the same notations and references.

We first recall briefly Specht's construction, using Macdonald's book as a reference [14, I, Appendix B].

Specht's construction. Let $G$ be a finite group, let $G_{*}$ be the set of conjugacy classes in $G$ and let $G^{*}$ be the set of irreducible characters of $G$. Given a conjugacy class $c \in G_{*}$, we denote by $\zeta_{c}$ the order of the centralizer of an element of $c$. We denote the value of a character $\gamma$ of $G$ at any element of a comjugacy class $c \in G_{*}$ by $\gamma(c)$.

We denote the wreath product $G>\mathfrak{S}_{n}$ by $G_{n}$. This wreath product is the semidirect product $G^{n} \rtimes \mathfrak{S}_{n}$ for the action of $\mathfrak{S}_{n}$ on $G^{n}$ given by

$$
\sigma \cdot\left(g_{1}, \ldots, g_{n}\right)=\left(g_{\sigma^{-1}(1)}, \ldots, g_{\sigma^{-1}(n)}\right)
$$

where $\sigma \in \mathfrak{S}_{n}$ and $\left(g_{1}, \ldots, g_{n}\right) \in G^{n}$, so that we can always represent an element in $G_{n}$ as a product $\left(g_{1} \ldots, g_{n}\right) \sigma$.

Given a complex representation $V$ of $G$, we construct a complex representation $\eta_{n}(V)$ of $G_{n}$ on the space $V^{\otimes n}$ by letting a product $\left(g_{1} \ldots, g_{n}\right) \sigma$ acting on a pure tensor $v_{1} \otimes \cdots \otimes v_{n} \in V^{\otimes n}$ in the following way:

$$
\left(\left(g_{1} \ldots, g_{n}\right) \sigma\right) \cdot\left(v_{1} \otimes \cdots \otimes v_{n}\right)=\left(g_{1} \cdot v_{\sigma^{-1}(1)}\right) \otimes \cdots \otimes\left(g_{n} \cdot v_{\sigma^{-1}(n)}\right) .
$$

The character of $\eta_{n}(V)$ does not depend on $V$ but only of its character; if $\rho$ denotes the latter, then we will denote the former by $\eta_{n}(\rho)$.

We let $\mathcal{P}$ be the set of all partitions, and we set $\mathcal{P}_{G}=\mathcal{P}^{G^{*}}$. Given an element $\lambda=\left(\lambda_{\gamma}\right)_{\gamma \in G^{*}}$ in $\mathcal{P}_{G}$, we denote by $|\lambda|$ the sum $\sum_{\gamma}\left|\lambda_{\gamma}\right|$.

Now let $\Lambda_{\mathbb{C}}$ be the (free) ring of symmetric polynomials with complex coefficients. As is well-known, $\Lambda_{\mathbb{C}}$ is generated over $\mathbb{C}$ by a countable family of algebraically independant elements: one can choose for generators the family $\left(h_{n}\right)_{n \geq 1}$ of complete symmetric functions or the family $\left(p_{n}\right)_{n \geq 1}$ of power sums. On the other hand, the family of Schur functions $\left(s_{\lambda}\right)_{\lambda_{\in} \mathcal{P}}$ is a basis of the vector space $\Lambda_{\mathbb{C}}$. Following Macdonald, we denote by $\Lambda_{\mathbb{C}}(G)$ the ring of polynomials over $\mathbb{C}$ in the family of variables $\left(p_{n}(c)\right)_{n \geq 1, c \in G_{*}}$. Setting

$$
p_{n}(\gamma)=\sum_{c \in G_{*}} \zeta_{c}^{-1} \gamma(c) p_{r}(c)
$$

for any $\gamma \in G^{*}$, one can easily check that $\Lambda_{\mathbb{C}}(G)$ is also the ring of polynomials in the variables $\left(p_{n}(\gamma)\right)_{n \geq 1, \gamma \in G^{*}}$. Every symmetric polynomial $P \in \Lambda_{\mathbb{C}}$ can be expressed as a polynomial with complex coefficients in the power sums $p_{n}$; given $\gamma \in G^{*}$, we denote by $P(\gamma)$ the element of $\Lambda_{\mathbb{C}}(G)$ obtained by replacing the variables $p_{n}$ by the 
variables $p_{n}(\gamma)$ in the expression of $P$. Given an element $\lambda=\left(\lambda_{\gamma}\right)_{\gamma \in G^{*}}$ in $\mathcal{P}_{G}$, we set

$$
s_{\lambda}=\prod_{\gamma \in G^{*}} s_{\lambda_{\gamma}}(\gamma)
$$

The set of complex irreducible characters of $G_{n}$ is a basis of the algebra of complex-valued class functions of $G_{n}$, so that we can denote this latter by $\mathbb{C} \operatorname{Irr}\left(G_{n}\right)$. The direct sum

$$
R(G)=\bigoplus_{n \geq 0} \mathbb{C} \operatorname{Irr}\left(G_{n}\right)
$$

can then be endowed with the structure of a commutative and cocommutative $\mathbb{N}$-graded Hopf algebra, where the product is given by (the maps induced on the level of characters by) the induction functors $\operatorname{Ind}_{G_{m} \times G_{n}}^{G_{m+n}}$ and the coproduct is afforded likewise by the restriction functors $\operatorname{Res}_{G_{m} \times G_{n}}^{G_{m+n}}$, 14, I, Appendix B, 4 and I, 7, Example 26]. Since $\Lambda_{\mathbb{C}}(G)$ is a free commutative algebra, there is a unique homomorphism of $\mathbb{C}$-algebras

$$
\operatorname{ch}^{-1}: \Lambda_{\mathbb{C}}(G) \rightarrow R(G)
$$

with the following property: for each $n \geq 0$ and each $c \in G_{*}, \mathrm{ch}^{-1}$ maps the variable $p_{n}(c)$ to the characteristic function of the conjugacy class of $G_{n}$ consisting of the products $\left(g_{1}, \ldots, g_{n}\right) \sigma$, where the permutation $\sigma \in \mathfrak{S}_{n}$ is a $n$-cycle and the product $g_{1} g_{2} \cdots g_{n}$ belongs to the conjugacy class $c$. It turns out that $\mathrm{ch}^{-1}$ is an isomorphism of Hopf algebra, whose inverse will be denoted by ch. Then, using arguments of orthogonality and integrality, it can be shown [14] I, Appendix B, 9] that the image under ch of the irreducible characters of $G_{n}$ are the elements $s_{\lambda}$, where $\lambda \in \mathcal{P}_{G}$ is such that $|\lambda|=n$.

Later on, we will need to know the image under ch of characters $\eta_{n}(\rho)$. We do the computation now.

Lemma 6.1. Let $\gamma_{1}, \ldots, \gamma_{s}$ the irreducible characters of $G$, let $c_{1}, \ldots, c_{s}$ be non-negative integers, and set $\rho=c_{1} \gamma_{1}+\cdots+c_{s} \gamma_{s}$. Then

$$
\sum_{n \geq 0} \operatorname{ch}\left(\eta_{n}(\rho)\right)=\prod_{i=1}^{s}\left(\sum_{n \geq 0} h_{n}\left(\gamma_{i}\right)\right)^{c_{i}} .
$$

Proof. The proof given in [14 I, Appendix B, 8] for the case where $\rho$ is irreducible can be easily adapted. Indeed in the computation that follows Equation (8.2) in that reference, the steps that lead to the equality

$$
\sum_{n \geq 0} \operatorname{ch}\left(\eta_{n}(\gamma)\right)=\exp \left(\sum_{r \geq 1} \frac{1}{r} \sum_{c \in G_{*}} \zeta_{c}^{-1} \gamma(c) p_{r}(c)\right)
$$


are valid even if the character $\gamma$ is reducible. Applying this formula to the character $\rho$, we get

$$
\begin{aligned}
\sum_{n \geq 0} \operatorname{ch}\left(\eta_{n}(\rho)\right) & =\exp \left(\sum_{r \geq 1} \frac{1}{r} \sum_{c \in G_{*}} \zeta_{c}^{-1} \rho(c) p_{r}(c)\right) \\
& =\prod_{i=1}^{s}\left[\exp \left(\sum_{r \geq 1} \frac{1}{r} \sum_{c \in G_{*}} \zeta_{c}^{-1} \gamma_{i}(c) p_{r}(c)\right)\right]^{c_{i}} \\
& =\prod_{i=1}^{s}\left[\exp \left(\sum_{r \geq 1} \frac{1}{r} p_{r}\left(\gamma_{i}\right)\right)\right]^{c_{i}} \\
& =\prod_{i=1}^{s}\left[\sum_{n \geq 0} h_{n}\left(\gamma_{i}\right)\right]^{c_{i}},
\end{aligned}
$$

the last step in the computation coming from Newton's formulas.

The comparison result. Having now recalled Specht's construction of the irreducible characters for the wreath product $G \prec \mathfrak{S}_{n}$ of an arbitrary finite group $G$ by the symmetric group $\mathfrak{S}_{n}$, we can specialize to the case where $G$ is the group $W=\mathbb{Z} / 2 \mathbb{Z}$ with two elements. The notation $W_{n}$ for the wreath product $W$ $\mathfrak{S}_{n}$ then agrees with its use by Bonnafé and Hohlweg. The Hopf algebra $R(W)$ is identical to the complexified Hopf algebra $\mathcal{C H} \mathcal{A R} \otimes_{\mathbb{Z}} \mathbb{C}$. The set $W^{*}$ of irreducible characters of $W$ has two elements, namely the trivial character $\tau$ and the signature $\varepsilon$. One can view an element $\lambda=\left(\lambda_{\tau}, \lambda_{\varepsilon}\right)$ of $\mathcal{P}_{W}$ as a bipartition $\left(\lambda^{+}, \lambda^{-}\right)$by setting $\lambda^{+}=\lambda_{\tau}$ and $\lambda^{-}=\lambda_{\varepsilon}$. As a final piece of notation, we set $\lambda^{*}=\left(\lambda^{+},\left(\lambda^{-}\right)^{t}\right)$ for any bipartition $\lambda=\left(\lambda^{+}, \lambda^{-}\right)$.

Generalizing Poirier and Reutenauer's work [17 for symmetric groups to the case of $W_{n}$, we define a linear map:

$$
f: \mathcal{Q} \otimes_{\mathbb{Z}} \mathbb{C} \longrightarrow \Lambda_{\mathbb{C}}(W)
$$

by setting $f\left(z_{Q}\right)=s_{(\operatorname{sh} Q) *}$ for any bitableau $Q$. With all these notations, our result can be stated as follows:

Theorem 6.2. The following diagram of $\mathbb{N}$-graded Hopf algebras

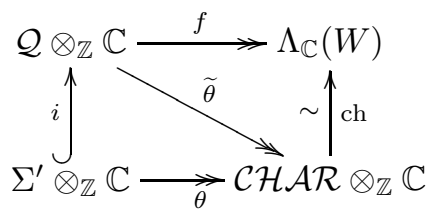

is commutative. In particular $\operatorname{ch}\left(\xi_{\lambda}\right)=s_{\lambda^{*}}$, for any bipartition $\lambda$, so that Bonnafé and Hohlweg's construction is equivalent to Specht's one, up to a relabelling.

Some further notation and a bijection will be needed for the proof. We present them now. 
Some notations and a bijection. We call quasicomposition a sequence $E=$ $\left(e_{1}, e_{2}, e_{3}, \ldots\right)$ of non-negative integers, all of whose terms but a finite number vanish. The size $|E|$ of $E$ is the sum $e_{1}+e_{2}+e_{3}+\cdots$ of the terms. Given a partition $\mu$ and a quasicomposition $E$, we denote by $\operatorname{Tab}(\mu, E)$ the set of all semistandard tableau of shape $\mu$ and weight $E$, that is the set of all fillings of the Ferrers diagram of shape $\mu$ with positive integers, in such a way that the numbers are weakly increasing from left to right in the rows, strictly increasing from top to bottom in the columns, and that there is $e_{1}$ times the number $1, e_{2}$ times the number 2, and so on [14 p. 5]. The set $\operatorname{Tab}(\mu, E)$ is of course empty unless $|\mu|=|E|$. Given any quasicomposition $E=\left(e_{1}, e_{2}, e_{3}, \ldots\right)$, the formula

$$
h_{e_{1}} h_{e_{2}} h_{e_{3}} \cdots=\sum_{\mu \in \mathcal{P}}|\operatorname{Tab}(\mu, E)| s_{\mu}
$$

holds in $\Lambda_{\mathbb{C}}$ (see [14, I, (6.4)] for a proof).

Now we fix a positive integer $n$ and a signed composition $C=\left(c_{1}, \ldots, c_{\ell}\right)$ of it. Let $\ell$ be the length of $C$. We define $\operatorname{Comp}(C)$ as the set of all quasicompositions $D=\left(d_{1}, \ldots, d_{\ell}\right)$ such that $d_{i}=0$ if $c_{i}>0$ and $0 \leq d_{i} \leq-c_{i}$ if $c_{i}<0$. Given such a $D$, we further define two quasicompositions $T_{C, D}=\left(t_{1}, \ldots, t_{\ell}\right)$ and $E_{C, D}=$ $\left(e_{1}, \ldots, e_{\ell}\right)$ by

$$
t_{i}=\left\{\begin{array}{ll}
c_{i} & \text { if } c_{i}>0, \\
d_{i} & \text { if } c_{i}<0 ;
\end{array} \text { and } \quad e_{i}= \begin{cases}0 & \text { if } c_{i}>0, \\
-c_{i}-d_{i} & \text { if } c_{i}<0 .\end{cases}\right.
$$

The signed composition obtained by omitting the zeros in the list

$$
\left(-e_{1}, t_{1},-e_{2}, t_{2}, \ldots,-e_{\ell}, t_{\ell}\right)
$$

will be denoted by $B_{C, D}$. For instance, for $C=(2, \overline{2}, \overline{3}, 1, \overline{1}, 2,2, \overline{2}) \|=15$, we can choose $D=(0,0,2,0,1,0,0,0)$, and then $T_{C, D}=(2,0,2,1,1,2,2,0), E_{C, D}=$ $(0,2,1,0,0,0,0,2)$ and $B_{C, D}=(2, \overline{2}, \overline{1}, 2,1,1,2,2, \overline{2})$.

Finally, given a bipartition $\lambda=\left(\lambda^{+}, \lambda^{-}\right)$and a signed composition $C$ with $|\lambda|=$ $|C|$, we define $\operatorname{Bitab}(\lambda, C)$ as the set of all standard bitableaux $Q$ such that $\operatorname{sh}(Q)=$ $\lambda^{*}$ and $C \leftarrow \mathbf{C}(Q)$ (see Remark 4.7).

One of the key to the proof of Theorem 6.2 is the following combinatorial result.

Proposition 6.3. Given a bipartition $\lambda$ and a signed composition $C$ with $|\lambda|=|C|$, the sets $\operatorname{Bitab}(\lambda, C)$ and

$$
\coprod_{D \in \operatorname{Comp}(C)} \operatorname{Tab}\left(\lambda^{+}, T_{C, D}\right) \times \operatorname{Tab}\left(\lambda^{-}, E_{C, D}\right)
$$

have the same cardinality.

Proof. Let $n$ be a positive integer, $C$ be a signed composition of $n$, and $\lambda=\left(\lambda^{+}, \lambda^{-}\right)$ be a bipartition with $|\lambda|=n$. We construct inverse bijections between $\operatorname{Bitab}(\lambda, C)$ and

$$
\coprod_{D \in \operatorname{Comp}(C)} \operatorname{Tab}\left(\lambda^{+}, T_{C, D}\right) \times \operatorname{Tab}\left(\lambda^{-}, E_{C, D}\right)
$$

as follows.

First let $(R, S)$ be in the second set, so that $R \in \operatorname{Tab}\left(\lambda^{+}, T_{C, D}\right)$ and $S \in$ $\operatorname{Tab}\left(\lambda^{-}, E_{C, D}\right)$ for some $D \in \operatorname{Comp}(C)$. We can put a total order on the boxes in $R$ and $S$ by requiring that: 
- A box is smaller than another one if the label written in it is smaller than the one in the other.

- Given two boxes with the same label in it, a box in $S$ is smaller than a box in $R$.

- For boxes containing the same label and located in the same tableau ( $R$ or $S$ ), boxes located south-west are smaller than boxes located north-east.

We can then enumerate in increasing order the boxes in $R$ and $S$. Filling now each box of $R$ and $S$ by its rank of appearance in the enumeration, we construct a standard bitableau $\tilde{Q}$ of shape $\lambda$. We then define $Q$ as the bitableau obtained from $\tilde{Q}$ by transposing $\tilde{Q}^{-}$, so that $Q$ has shape $\lambda^{*}$. Comparing this construction with the combinatorial rule in Remark 4.7 that computes $\boldsymbol{C}(Q)$, we easily check that the signed composition $B_{C, D}$ can be obtained from $\mathbf{C}(Q)$ by refinement of the parts, so that $C \stackrel{B}{\longleftarrow} B_{C, D} \stackrel{R}{\longleftarrow} \mathbf{C}(Q)$, which implies $Q \in \operatorname{Bitab}(\lambda, C)$.

In the other direction, let $Q$ be a given element in $\operatorname{Bitab}(\lambda, C)$. From Theorem 3.15 there exists a unique signed composition $B$ such that $C \stackrel{B}{\longleftarrow} B \stackrel{R}{\longleftarrow} \mathbf{C}(Q)$, and we can find a (unique) element $D \in \operatorname{Comp}(C)$ so that $B=B_{C, D}$. Now we transpose $Q^{-}$and get a bitableau $\tilde{Q}$. We construct a list $L=\left(l_{1}, l_{2}, \ldots, l_{n}\right)$ of positive integers by placing first $\left|c_{1}\right|$ times the number 1 , then $\left|c_{2}\right|$ times the number 2 , and so on. Then we substitute $l_{1}$ to $1, l_{2}$ to 2 , and so on, in the boxes of $\tilde{Q}$, and obtain in this way a pair $(R, S)$ of tableaux of shapes $\lambda^{+}$and $\lambda^{-}$respectively. The fact that $B_{C, D} \stackrel{R}{\longleftarrow} \mathbf{C}(Q)$ implies that this construction yield two semistandard tableaux $R$ and $S$ with weights $T_{C, D}$ and $E_{C, D}$ respectively, that is to say

$$
(R, S) \in \operatorname{Tab}\left(\lambda^{+}, T_{C, D}\right) \times \operatorname{Tab}\left(\lambda^{-}, E_{C, D}\right) .
$$

It is a routine task to check that the two above constructions yield mutually inverse bijections between $\coprod_{D \in \operatorname{Comp}(C)} \operatorname{Tab}\left(\lambda^{+}, T_{C, D}\right) \times \operatorname{Tab}\left(\lambda^{-}, E_{C, D}\right)$ and $\operatorname{Bitab}(\lambda, C)$.

We end this paragraph by an example that illustrates the constructions needed in the proof above. We take $n=15$ and choose the same signed composition $C$ as in the previous example, namely

$$
C=(2, \overline{2}, \overline{3}, 1, \overline{1}, 2,2, \overline{2}) .
$$

We choose $\lambda^{+}=631$ and $\lambda^{-}=41$, so that $\lambda^{*}=\left(631,21^{3}\right)$. Starting from the pair $(R, S)$ with

$$
R=\begin{array}{|l|l|l|l|l|l}
\hline 1 & 1 & 3 & 3 & 4 & 7 \\
\hline 5 & 6 & 7 & &
\end{array} \quad \text { and } \quad S=\begin{array}{|l|l|l|l|}
2 & 2 & 3 & 8 \\
\hline 6 & &
\end{array}
$$

we construct $\tilde{Q}=\left(\tilde{Q}^{+}, \tilde{Q}^{-}\right)$where

$$
\tilde{Q}^{+}=\begin{array}{|l|l|l|l|l|l}
\hline 1 & 2 & 6 & 7 & 8 & 13 \\
\hline 9 & 11 & 12 & &
\end{array} \quad \text { and } \quad \tilde{Q}^{-}=\begin{array}{|l|l|l|l|}
\hline 3 & 4 & 5 & 15 \\
\hline 10 & 14 & &
\end{array}
$$

whence $Q=\left(Q^{+}, Q^{-}\right)$with

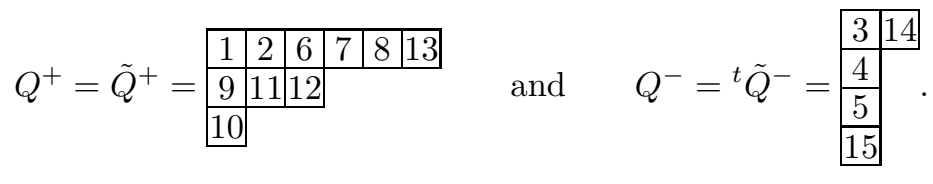


Since $\mathbf{C}(Q)=(2, \overline{3}, 3,1,4, \overline{2})$, it holds that $C \stackrel{B}{\longleftarrow} B \stackrel{R}{\longleftarrow} \mathbf{C}(Q)$ with

$$
B=(2, \overline{2}, \overline{1}, 2,1,1,2,2, \overline{2}) \text {, }
$$

which implies $C \leftarrow \mathbf{C}(Q)$.

In the other direction, we start from the bitableau $Q$. We observe that the signed composition $B$ such that $C \stackrel{B}{\longleftarrow} B \stackrel{R}{\longleftarrow} \mathbf{C}(Q)$ is $B_{C, D}$, where $D$ is given by $D=(0,0,2,0,1,0,0,0)$. Now we write down the list

$$
L=(1,1,2,2,3,3,3,4,5,6,6,7,7,8,8)
$$

from $C$. Transposing the negative tableau $Q^{-}$, we write down $\tilde{Q}$ and substitute the elements of $L$ to the numbers in the boxes of $\tilde{Q}$. We recover our original pair $(R, S)$. We easily verify that $R$ has weight

$$
T_{C, D}=(2,0,2,1,1,2,2,0)
$$

and that $S$ has weight

$$
E_{C, D}=(0,2,1,0,0,0,0,2) .
$$

\section{Proof of Theorem 6.2}

1 We first compute the image by ch of the induced character $\operatorname{Ind}_{\mathfrak{S}_{n}}^{W_{n}} 1$ of $W_{n}$, where $n$ is a positive integer. To do that, we construct the complex representation $\eta_{n}(V)$ of $W_{n}$, where $V$ is the left regular representation of $W=\mathbb{Z} / 2 \mathbb{Z}$. Denoting by $\mathbb{C}_{1}$ the trivial representation of $\mathfrak{S}_{n}$, we then observe that the isomorphism of vector spaces $\operatorname{Ind}_{\mathfrak{S}_{n}}^{W_{n}} \mathbb{C}_{1} \cong \eta_{n}(V)$ given by the sequence of natural identifications

$$
\operatorname{Ind}_{\mathfrak{S}_{n}}^{W_{n}} \mathbb{C}_{1} \cong \mathbb{C} W_{n} \otimes \mathbb{C S}_{n} \mathbb{C}_{1} \cong \mathbb{C}\left(W^{n}\right) \cong(\mathbb{C} W)^{\otimes n}=V^{\otimes n}=\eta_{n}(V)
$$

is compatible with the action of $W_{n}$. Since $V$ has $\tau+\varepsilon$ for character, it follows that $\operatorname{Ind}_{\mathfrak{S}_{n}}^{W_{n}} 1=\eta_{n}(\tau+\varepsilon)$. Lemma 6.1 now implies that

$$
\sum_{n \geq 0} \operatorname{ch}\left(\operatorname{Ind}_{\mathfrak{S}_{n}}^{W_{n}} 1\right)=\left(\sum_{n \geq 0} h_{n}(\tau)\right)\left(\sum_{n \geq 0} h_{n}(\varepsilon)\right) .
$$

On the other side, it is easy to check that $\eta_{n}(\tau)$ is the trivial character of $W_{n}$. Therefore ch maps the trivial character $\operatorname{Ind}_{W_{n}}^{W_{n}} 1$ of $W_{n}$ to $h_{n}(\tau)$. To comply with the philosophy used by Bonnafé and Hohlweg, we will write for any positive integer $n$

$$
\varphi_{ \pm n}=\operatorname{ch}\left(\operatorname{Ind}_{W_{ \pm n}}^{W_{n}} 1\right)= \begin{cases}\operatorname{ch}\left(\operatorname{Ind}_{W_{n}}^{W_{n}} 1\right)=h_{n}(\tau) & \text { for '+' sign, } \\ \operatorname{ch}\left(\operatorname{Ind}_{\mathfrak{S}_{n}}^{W_{n}} 1\right)=\sum_{k=0}^{n} h_{k}(\tau) h_{n-k}(\varepsilon) & \text { for '-' sign. }\end{cases}
$$

2 We now prove the equality $f \circ i=\operatorname{ch} \circ \theta$. Given any signed composition $C=$ $\left(c_{1}, \ldots, c_{\ell}\right)$, there holds $x_{C}=x_{c_{1}} \cdots x_{c_{\ell}}$. Since $\theta$ is a morphism of Hopf algebras, we can write

$$
\operatorname{Ind}_{W_{C}}^{W_{|C|}} 1_{C}=\theta\left(x_{C}\right)=\theta\left(x_{c_{1}}\right) \cdots \theta\left(x_{c_{\ell}}\right)=\operatorname{Ind}_{W_{c_{1}}}^{W_{\left|c_{1}\right|}} 1 \bullet \cdots \bullet \operatorname{Ind}_{W_{c_{\ell}}}^{W_{\left|c_{\ell}\right|}} 1,
$$

and taking its image under ch,

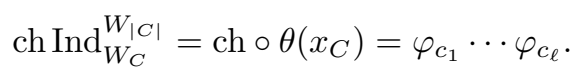

The formula

$$
\varphi_{-n}=\sum_{k=0}^{n} h_{k}(\tau) h_{n-k}(\varepsilon)
$$


valid for any positive integer $n$, makes possible to continue the computation:

$$
\operatorname{ch} \circ \theta\left(x_{C}\right)=\sum_{D \in \operatorname{Comp}(C)} h_{t_{1}}(\tau) \cdots h_{t_{\ell}}(\tau) h_{e_{1}}(\varepsilon) \cdots h_{e_{\ell}}(\varepsilon),
$$

where the quasicompositions $\left(t_{1}, \ldots, t_{\ell}\right)$ and $\left(e_{1}, \ldots, e_{\ell}\right)$ appearing in the sum are $T_{C, D}$ and $E_{C, D}$ respectively. We thus get, using Proposition 6.3 and the decomposition of $X_{C}$ given at the end of Remark 4.7.

$$
\begin{aligned}
\operatorname{ch} \circ \theta\left(x_{C}\right) & =\sum_{D \in \operatorname{Comp}(C)}\left(\sum_{\lambda^{+} \in \mathcal{P}}\left|\operatorname{Tab}\left(\lambda^{+}, T_{C, D}\right)\right| s_{\lambda^{+}}(\tau)\right)\left(\sum_{\lambda^{-} \in \mathcal{P}}\left|\operatorname{Tab}\left(\lambda^{-}, E_{C, D}\right)\right| s_{\lambda^{-}}(\varepsilon)\right) \\
& =\sum_{\left(\lambda^{+}, \lambda^{-}\right) \in \mathcal{P}_{W}}\left(\sum_{D \in \operatorname{Comp}(C)}\left|\operatorname{Tab}\left(\lambda^{+}, T_{C, D}\right) \times \operatorname{Tab}\left(\lambda^{-}, E_{C, D}\right)\right|\right) s_{\lambda^{+}}(\tau) s_{\lambda^{-}}(\varepsilon) \\
& =\sum_{\lambda \in \mathcal{P}_{W}}|\operatorname{Bitab}(\lambda, C)| s_{\lambda} \\
& =\sum_{\substack{Q \text { std. bitableau } \\
C \leftarrow C(Q)}} s_{(\operatorname{sh} Q)^{*}} \\
& =\sum_{\substack{Q \text { std. bitableau } \\
C \leftarrow \boldsymbol{C}(Q)}} f\left(z_{Q}\right) \\
& =f \circ i\left(x_{C}\right) .
\end{aligned}
$$

Since the elements $x_{C}$ generate $\Sigma^{\prime} \otimes_{\mathbb{Z}} \mathbb{C}$ as a vector space, it follows that $\operatorname{ch} \circ \theta=f \circ i$.

3 To complete the proof, it now suffices to show that $f=\operatorname{ch} \circ \tilde{\theta}$. We first observe that both members of this equality coincide on the image of $i$ in $\mathcal{Q} \otimes_{\mathbb{Z}} \mathbb{C}$, since

$$
\operatorname{ch} \circ \theta=f \circ i \quad \text { and } \quad \theta=\tilde{\theta} \circ i \text {. }
$$

On the other hand, $f$ and cho $\tilde{\theta}$ have the same kernel, namely the vector space $\mathcal{Q}_{n}^{\perp}$ spanned by the elements $z_{Q}-z_{Q^{\prime}}$ for standard bitableaux $Q$ and $Q^{\prime}$ of the same shape (see Theorem 4.14). Since $\theta$ is surjective, this kernel, together with the image of $i$, spans $\mathcal{Q} \otimes_{\mathbb{Z}} \mathbb{C}$. The result follows easily from these facts. 